\title{
Characterizing reinforcement learning methods through parameterized learning problems
}

\author{
Shivaram Kalyanakrishnan • Peter Stone
}

Received: 6 March 2010 / Accepted: 4 May 2011 / Published online: 3 June 2011

(C) The Author(s) 2011

\begin{abstract}
The field of reinforcement learning (RL) has been energized in the past few decades by elegant theoretical results indicating under what conditions, and how quickly, certain algorithms are guaranteed to converge to optimal policies. However, in practical problems, these conditions are seldom met. When we cannot achieve optimality, the performance of RL algorithms must be measured empirically. Consequently, in order to meaningfully differentiate learning methods, it becomes necessary to characterize their performance on different problems, taking into account factors such as state estimation, exploration, function approximation, and constraints on computation and memory. To this end, we propose parameterized learning problems, in which such factors can be controlled systematically and their effects on learning methods characterized through targeted studies. Apart from providing very precise control of the parameters that affect learning, our parameterized learning problems enable benchmarking against optimal behavior; their relatively small sizes facilitate extensive experimentation.

Based on a survey of existing RL applications, in this article, we focus our attention on two predominant, "first order" factors: partial observability and function approximation. We design an appropriate parameterized learning problem, through which we compare two qualitatively distinct classes of algorithms: on-line value function-based methods and policy search methods. Empirical comparisons among various methods within each of these classes project $\operatorname{Sarsa}(\lambda)$ and Q-learning $(\lambda)$ as winners among the former, and CMA-ES as the winner in the latter. Comparing $\operatorname{Sarsa}(\lambda)$ and CMA-ES further on relevant problem instances, our study highlights regions of the problem space favoring their contrasting approaches. Short run-times for our experiments allow for an extensive search procedure that provides additional insights on relationships between method-specific parameters—such as eligibility traces, initial weights, and population sizes—and problem instances.
\end{abstract}

Editors: S. Whiteson and M. Littman.

S. Kalyanakrishnan $(\bowtie) \cdot$ P. Stone

Department of Computer Science, The University of Texas at Austin, 1616 Guadalupe St Suite 2.408, Austin, TX 78701, USA

e-mail: shivaram@cs.utexas.edu

P. Stone

e-mail: pstone@cs.utexas.edu 
Keywords Reinforcement learning · Empirical evaluation · Partial observability · Function approximation $\cdot$ Parameterized learning problem

\section{Introduction}

Sequential decision making from experience, or reinforcement learning (RL), is a wellsuited paradigm for agents seeking to optimize long-term gains as they carry out sensing, decision and action in an unknown environment. RL tasks are commonly formulated as Markov Decision Problems (MDPs). The solution of MDPs has benefited immensely from a strong theoretical framework that has been developed over the years. The cornerstone of this framework is the value function of the MDP (Bellman 1957), which encapsulates the longterm utilities of decisions. Control policies can be suitably derived from value functions; indeed several algorithms provably converge to optimal policies in finite MDPs (Watkins and Dayan 1992; Singh et al. 2000). Also, near-optimal behavior can be achieved after collecting a number of samples that is polynomial in the size of the state space $(|S|)$ and the number of actions $(|A|)$ (Kearns and Singh 2002; Brafman and Tennenholtz 2003; Strehl and Littman 2005), using a memory bounded in size by $O(|S||A|)$ (Strehl et al. 2006).

Unfortunately a large section of the RL tasks we face in the real world cannot be modeled and solved exactly as finite MDPs. Not only are the traditional objectives of convergence and optimality thereby inapplicable to a predominant number of tasks occurring in practice, in many of these tasks we cannot even ascertain the best performance that can be achieved, or how much training is necessary to achieve given levels of performance. The objective of learning in such practical tasks, which fall beyond the reach of current theoretical modeling, has to be rescaled to realizing policies with "high" expected long-term reward in a "sample efficient" manner, as determined empirically.

In a formal sense, the "No Free Lunch" theorems of Wolpert and Macready (1997) establish that for any optimization algorithm, an elevated performance in one class of problems is offset by worse performance in some other class. Even so, the enterprise of machine learning rests on the assumption that classes of problems encountered in practice tend to possess regularities, which can be actively characterized and exploited. Consequently, to the extent that the relationships between problem instances and the performance properties of algorithms are unclear, it becomes a worthwhile pursuit to uncover them. The need for such research has long been advocated: in an early editorial in this journal, Langley (1988, see p. 7) writes:

"For instance, one might find that learning method A performs better than method

$\mathrm{B}$ in one environment, whereas B fares better than A in another. Alternatively, one

might find interactions between two components of a learning method or two domain characteristics. We believe the most unexpected and interesting empirical results in machine learning will take this form."

The practice of supervised learning has benefitted from a number of empirical studies that seek to identify the strengths and weaknesses of learning methods. For example, Caruana and Niculescu-Mizil (2006) undertake a detailed comparison involving a number of supervised learning methods, test problems, and evaluation metrics. Caruana et al. (2008) present empirical results demonstrating that random forests (Breiman 2001) are typically more effective than several other classification methods on problems with high dimensionality (greater than 4000). Although the canonical boosting algorithm (Freund and Schapire 1996) enjoys desirable theoretical properties and is predominantly effective in practice, studies comparing it with other ensemble schemes such as bagging (Quinlan 1996; 
Bauer and Kohavi 1999) hint at its vulnerability in the presence of noisy training data. Banko and Brill (2001) advance the case that for problems with very large data sets (for example, natural language applications on the Internet), simple classifiers such as Winnow (Littlestone 1987) can be the most effective, and that voting-based ensemble schemes do not retain their attractiveness.

By associating problem characteristics with the strengths and weaknesses of supervised learning methods, the studies listed above provide useful "rules of thumb" to a practitioner who must choose a method to apply to a problem. Unfortunately the complex scope of the RL problem leaves the practitioner of RL with few such guidelines. Faced with a sequential decision making problem, not only does a designer need to pick a learning algorithm; he/she has to address the related issues of state estimation, exploration, and function approximation, while possibly satisfying computational and memory constraints. The broad motivation for this article is the eventual development of a "field guide" for the practice of RL, which would both inform the choices made by designers of RL solutions, and identify promising directions for future research.

Ultimately, a field guide would be evaluated based on the extent to which it can expedite the process of designing solutions for full-scale deployed applications. However, such applications are themselves too complex and constrained to provide reliable data from which the principles for a field guide can be inferred. Rather, there is a need for simpler, more transparent problems through which we, as designers, can systematically sort through the complex space of interactions between RL problems and solution strategies. This article joins a growing line of research in this direction (Moriarty et al. 1999; Gomez et al. 2008; Heidrich-Meisner and Igel 2008a; Whiteson et al. 2010).

The primary thrust of existing work on the subject has been in comparing RL algorithms on standard, benchmarking tasks, with possibly a small number of variations. By contrast, we design a synthetic, parameterized learning problem $^{1}$ with the explicit purpose of ascertaining the "working regions" of learning algorithms in a space that is carefully engineered to span the dimensions of the task and the learning architecture. The approach we propose enjoys the following merits:

1. The designed task and learning framework are easy to understand and can be controlled precisely.

2. We may examine the effect of subsets of problem parameters while keeping others fixed.

3. We can benchmark learned policies against optimal behavior.

4. The learning process can be executed in a relatively short duration of time, thereby facilitating extensive experimentation.

While the careful design of a synthetic learning problem allows us these liberties, equally it qualifies the extent to which our conclusions may generalize in practice. Thus, the results from our study are to be taken as starting points for further empirical investigation, rather than treated as well-grounded final products in themselves. In this sense, the methodology we put forth enjoys a complementary relationship with the research strategy of evaluating RL methods on more realistic problems. We proceed to demarcate the scope of our study.

\footnotetext{
${ }^{1}$ The term "parameterized learning problem" is quite generic; such problems have been used in the past both in RL and in other fields. For some examples, see our discussion of related work in Sect. 6. By applying the term here to describe our framework, we aim to underscore that problem parameters are its very crux; they are not secondary as in related work.
} 


\subsection{Scope of study}

In order to develop a field guide for solving realistic RL problems, it is first necessary to characterize such problems along the dimensions that distinguish them from well-understood cases such as table-based learning in finite MDPs. Towards this purpose, we undertake a survey of literature describing practical applications of RL. While surveying material from relevant journals and conference proceedings, we apply the criterion that the task application, rather than the learning method employed, be the primary focus of the publication. Based on our findings, we focus our attention on two predominant, "first order" factors that characterize a sizeable fraction of sequential decision making problems in practice: (a) partial observability, which arises due to an agent's inability to identify the system state, and (b) function approximation, which is necessary for learning in large or continuous state spaces. The ubiquity of these factors in applications is apparent from Table 1, which summarizes our survey. ${ }^{2}$

A majority of the applications listed in Table 1 have to contend with partial observability of state. In complex systems such as stock markets (Nevmyvaka et al. 2006), computer networks (Tesauro et al. 2007), and cellular tissue (Guez et al. 2008), available measurements seldom suffice to capture all the information that can affect decision making. Nearly every agent embedded in the real world (Kwok and Fox 2004; Ng et al. 2004; Lee et al. 2006) receives noisy sensory information. The inadequacy of the sensory signal in identifying the underlying system state hinders the assumption of a Markovian interaction between the agent and the environment, on which the theoretical guarantees associated with many learning methods rely. Whereas coping with partial observability in a systematic manner is a well-studied problem, it is yet to scale to complex tasks with large, high-dimensional, continuous state spaces (Chrisman 1992; Cassandra et al. 1994; Bakker et al. 2003; Pineau et al. 2006).

Of the 25 applications listed in Table 1, 15 involve continuous state spaces, which necessitate the use of function approximation in order to generalize. Indeed among the ten applications that have discrete state spaces, too, seven use some form of function approximation to represent the learned policy, as their state spaces are too large for enumeration, and possibly even infinite. The use of function approximation negates the theoretical guarantees of achieving optimal behavior. Often the function approximation scheme used is not capable of representing an optimal policy for a task; even when it is, seldom can it be proven that a learning algorithm will discover such a policy. Although there exist convergence guarantees for certain algorithms that use linear function approximation schemes (Konda and Tsitsiklis 2003; Perkins and Precup 2003; Maei et al. 2010), they do not provide effective lower bounds for the values of the learned policies. Further, convergence results rarely extend to situations in which non-linear representations such as neural networks are used to approximate the value function; yet non-linear representations are used commonly in practice, as apparent from Table 1.

Our survey of RL applications suggests that the most common strategy adopted while implementing sequential decision making in practice is to apply algorithms that come with provable guarantees under more restrictive assumptions, and to empirically verify

\footnotetext{
${ }^{2}$ Other independently-compiled surveys of sequential decision making applications corroborate the observations we draw based on Table 1. Langley and Pendrith (1998) describe several RL applications presented at a symposium organized around the topic; Szepesvári lists numerous applications from the control and approximate dynamic programming literature at this URL: http://www.ualberta.ca/ szepesva/RESEARCH/ RLApplications.html.
} 
Table 1 Characterization of some popular applications of reinforcement learning. "Policy Representation" describes the underlying representation from which the policy is derived. A "neural network" representation is non-linear, incorporating at least one hidden layer of units. Under tile coding, the number of "features" indicates the number of state variables, rather than the number of individual tiles

\begin{tabular}{|c|c|c|c|}
\hline Task & $\begin{array}{l}\text { State } \\
\text { observability }\end{array}$ & State space & $\begin{array}{l}\text { Policy representation } \\
\text { (number of features) }\end{array}$ \\
\hline $\begin{array}{l}\text { Backgammon } \\
\text { (Tesauro 1992) }\end{array}$ & Complete & Discrete & $\begin{array}{l}\text { Neural network } \\
\text { (198) }\end{array}$ \\
\hline $\begin{array}{l}\text { Job-shop scheduling } \\
\text { (Zhang and Dietterich 1995) }\end{array}$ & Complete & Discrete & $\begin{array}{l}\text { Neural network } \\
\text { (20) }\end{array}$ \\
\hline $\begin{array}{l}\text { Tetris } \\
\text { (Bertsekas and Tsitsiklis 1996) }\end{array}$ & Complete & Discrete & $\begin{array}{l}\text { Linear } \\
(21)\end{array}$ \\
\hline $\begin{array}{l}\text { Elevator dispatching } \\
\text { (Crites and Barto 1996) }\end{array}$ & Partial & Continuous & $\begin{array}{l}\text { Neural network } \\
\text { (46) }\end{array}$ \\
\hline $\begin{array}{l}\text { Acrobot control } \\
\text { (Sutton 1996) }\end{array}$ & Complete & Continuous & $\begin{array}{l}\text { Tile coding } \\
\text { (4) }\end{array}$ \\
\hline $\begin{array}{l}\text { Dynamic channel allocation } \\
\text { (Singh and Bertsekas 1997) }\end{array}$ & Complete & Discrete & $\begin{array}{l}\text { Linear } \\
\text { (100's) }\end{array}$ \\
\hline $\begin{array}{l}\text { Active guidance of finless rocket } \\
\text { (Gomez and Miikkulainen 2003) }\end{array}$ & Partial & Continuous & $\begin{array}{l}\text { Neural network } \\
\text { (14) }\end{array}$ \\
\hline $\begin{array}{l}\text { Fast quadrupedal locomotion } \\
\text { (Kohl and Stone 2004) }\end{array}$ & Partial & Continuous & $\begin{array}{l}\text { Parameterized policy } \\
\text { (12) }\end{array}$ \\
\hline $\begin{array}{l}\text { Robot sensing strategy } \\
\text { (Kwok and Fox 2004) }\end{array}$ & Partial & Continuous & $\begin{array}{l}\text { Linear } \\
(36)\end{array}$ \\
\hline $\begin{array}{l}\text { Helicopter control } \\
\text { (Ng et al. 2004) }\end{array}$ & Partial & Continuous & $\begin{array}{l}\text { Neural network } \\
\text { (10) }\end{array}$ \\
\hline $\begin{array}{l}\text { Dynamic bipedal locomotion } \\
\text { (Tedrake et al. 2004) }\end{array}$ & Partial & Continuous & $\begin{array}{l}\text { Feedback control policy } \\
\text { (2) }\end{array}$ \\
\hline $\begin{array}{l}\text { Adaptive job routing/scheduling } \\
\text { (Whiteson and Stone 2004) }\end{array}$ & Partial & Discrete & $\begin{array}{l}\text { Tabular } \\
\text { (4) }\end{array}$ \\
\hline $\begin{array}{l}\text { Robot soccer keepaway } \\
\text { (Stone et al. 2005) }\end{array}$ & Partial & Continuous & $\begin{array}{l}\text { Tile coding } \\
\text { (13) }\end{array}$ \\
\hline $\begin{array}{l}\text { Robot obstacle negotiation } \\
\text { (Lee et al. 2006) }\end{array}$ & Partial & Continuous & $\begin{array}{l}\text { Linear } \\
(10)\end{array}$ \\
\hline $\begin{array}{l}\text { Optimized trade execution } \\
\text { (Nevmyvaka et al. 2006) }\end{array}$ & Partial & Discrete & $\begin{array}{l}\text { Tabular } \\
(2-5)\end{array}$ \\
\hline $\begin{array}{l}\text { Blimp control } \\
\text { (Rottmann et al. 2007) }\end{array}$ & Partial & Continuous & $\begin{array}{l}\text { Gaussian process } \\
\text { (2) }\end{array}$ \\
\hline $\begin{array}{l}9 \times 9 \text { Go } \\
(\text { Silver et al. 2007) }\end{array}$ & Complete & Discrete & $\begin{array}{l}\text { Linear } \\
(\approx 1.5 \text { million })\end{array}$ \\
\hline $\begin{array}{l}\text { Ms. Pac-Man } \\
\text { (Szita and Lőrincz 2007) }\end{array}$ & Complete & Discrete & $\begin{array}{l}\text { Rule List } \\
\text { (10) }\end{array}$ \\
\hline $\begin{array}{l}\text { Autonomic resource allocation } \\
\text { (Tesauro et al. 2007) }\end{array}$ & Partial & Continuous & $\begin{array}{l}\text { Neural network } \\
\text { (2) }\end{array}$ \\
\hline
\end{tabular}


Table 1 (Continued)

\begin{tabular}{llll}
\hline Task & $\begin{array}{l}\text { State } \\
\text { observability }\end{array}$ & State space & $\begin{array}{l}\text { Policy representation } \\
\text { (number of features) }\end{array}$ \\
\hline $\begin{array}{l}\text { General game playing } \\
\text { (Finnsson and Björnsson 2008) }\end{array}$ & Complete & Discrete & $\begin{array}{l}\text { Tabular } \\
\text { (over part of state space) }\end{array}$ \\
$\begin{array}{l}\text { Soccer opponent "hassling" } \\
\text { (Gabel et al. 2009) }\end{array}$ & Partial & Continuous & $\begin{array}{l}\text { Neural network } \\
\text { Adaptive epilepsy treatment } \\
\text { (Guez et al. 2008) }\end{array}$ \\
$\begin{array}{l}\text { Computer memory scheduling } \\
\text { (Ípek et al. 2008) }\end{array}$ & Partial & Continuous & Extremely randomized trees \\
$\begin{array}{l}\text { Motor skills } \\
\text { (Peters and Schaal 2008) }\end{array}$ & Complete & Discrete & (114) \\
$\begin{array}{l}\text { Combustion Control } \\
\text { (Hansen et al. 2009) }\end{array}$ & Partial & Continuous & (6) \\
\hline
\end{tabular}

that they remain effective when those assumptions are relaxed. Typically much manual effort is expended in designing schemes to mitigate the adverse effects partial observability and inadequate function approximation. In addition recent lines of research have focused on developing adaptive methods to cope with these factors (Pineau et al. 2006; Whiteson and Stone 2006; Mahadevan 2009). While such methods can improve the performance of RL algorithms in practice, their effectiveness is yet to be demonstrated on a wide scale; it remains that even in the situations they apply, the undesirable effects of partial observability and function approximation are only reduced, and not eliminated.

Adopting the view that in practice, partial observability and function approximation will affect learning to varying degrees, we aim to examine the capabilities of learning methods that operate in their presence. Specifically we design a framework in which these factors can be systematically controlled to gauge their effect on different learning methods. While these factors can be construed as aspects of an agent's learning apparatus, our study also considers task-specific characteristics such as the size of the state space and the stochasticity of actions. Any fixed setting for the parameters that control these factors determines a learning problem, on which different learning methods can be compared.

In our study, we compare learning methods from two contrasting classes of algorithms. The first class corresponds to (model-free) on-line value function-based methods, which learn by associating utilities with action choices from individual states. The second class of algorithms we examine are policy search methods. Rather than learn a value function, policy search methods seek to directly optimize the parameters representing a policy, treating the expected long-term reward accrued as an objective function to maximize.

First we evaluate several methods within each of the above classes, and based on their empirical performance, pick one method from each class to further compare across a suite of problem instances. The representatives thus chosen are Sarsa $(\lambda)$ (Rummery and Niranjan 1994; Sutton and Barto 1998) from the class of on-line value function-based methods, and CMA-ES (Hansen 2009) from the class of policy search methods. In evaluating a method on a problem instance, our experimental framework allows us to extensively search for the method-specific parameters (such as learning rates, eligibility traces, and sample sizes for fitness evaluation) that lead to the method's best performance. Our experiments identify 
regions of the problem space that are better suited to on-line value function-based and policy search methods, and yield insights about the effect of algorithm-specific parameters.

The remainder of this article is organized as follows. In Sect. 2, we describe the detailed design of our parameterized learning problem. Section 3 provides brief descriptions of the methods compared in the study. In Sect. 4, we present detailed results from our experiments, which we follow with a discussion in Sect. 5. Related work is discussed in Sect. 6. We summarize and conclude the article in Sect. 7.

\section{A Parameterized sequential decision making problem}

In this section, we describe the construction of our parameterized learning problem, which is composed of a task MDP and an accompanying learning framework that incorporates partial observability and function approximation.

\subsection{Problem size and stochasticity}

The class of tasks we design consists of simple square grids, each having a finite number of states. An example of such a task is illustrated in Fig. 1. The size of the state space is $s^{2}-1$, where $s$, the side of the square, serves as a parameter to be varied. Each episode begins with the agent placed in a start state chosen uniformly at random from among the set of non-terminal states, as depicted in Fig. 1(a). The north and east sides of the grid are lined with terminal states, of which there are $2(s-1)$. From each state, the agent can take either of two actions: North $(\mathbf{N})$ and $\mathbf{E a s t}(\mathbf{E})$. On taking $\mathbf{N}(\mathbf{E})$, the agent moves north (east) with probability $p$ and it moves east (north) with probability $1-p$. The variable $p$, which essentially controls the stochasticity in the transitions, is also treated as a parameter of the task MDP. Note that irrespective of the value of $p$, the agent always moves either north or east on each transition before reaching a terminal state. Consequently episodes last at most $2 s-3$ steps.

Through the course of each episode, the agent accrues rewards at the states it visits. Each MDP is initialized with a fixed set of rewards drawn uniformly from $[0,1]$, as illustrated in Fig. 1(b). In general the rewards in an MDP can themselves be stochastic, but in our tests, we find that the effect of stochastic rewards on our learning algorithms is qualitatively similar to the effect of stochastic state transitions, which are controlled by the parameter $p$. Thus, we keep the rewards deterministic. Figures 1(c) and 1(d) show the optimal values

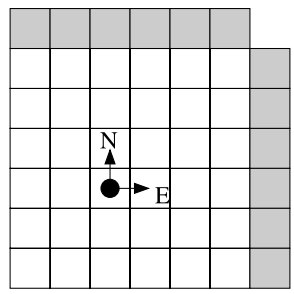

$\square$ Non-terminal $\square$ Terminal

(a)

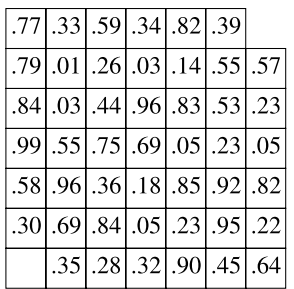

Example Rewards

(b)

\begin{tabular}{|c|c|c|c|c|c|c|}
\hline 0 & 0 & 0 & 0 & 0 & 0 & \\
\hline 1.2 & 1.3 & 1.1 & 1.1 & 1.1 & 0.6 & 0 \\
\hline 3.1 & 3.2 & 3.0 & 2.2 & 1.5 & 1.0 & 0 \\
\hline 4.8 & 4.4 & 3.7 & 3.1 & 2.3 & 1.4 & 0 \\
\hline 5.9 & 4.9 & 4.4 & 3.7 & 2.5 & 1.5 & 0 \\
\hline 6.5 & 5.8 & 4.7 & 3.8 & 3.3 & 2.2 & 0 \\
\cline { 1 - 5 } 6.8 & 6.4 & 5.5 & 4.4 & 3.5 & 2.9 & 0 \\
\cline { 1 - 2 } & & & & & &
\end{tabular}

Values of optimal actions

(c)

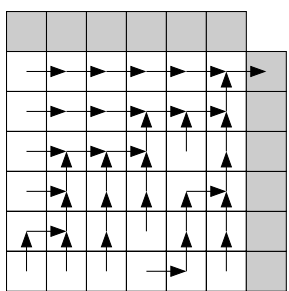

Optimal Policy $(\mathrm{p}=0.1)$

(d)

Fig. 1 (a) Example of parameterized MDP example with $s=7$; the number of non-terminal states is 36 . (b) Rewards obtained at "next states" of transitions. (c) Optimal action values from each state when $p=0.1$. (d) Corresponding optimal policy 
and the actions to which they correspond under the reward structure shown in Fig. 1(b) (assuming $p=0.1$ ). We do not discount rewards in the computation of values. Notice that the variation in values along the north and east directions is gradual: this supports the scope for generalization between neighboring cells. The values in Fig. 1(c) are obtained using dynamic programming. Indeed it is also straightforward under this setup to learn the optimal policy based on experience, for example by using a table of action values updated through Q-learning. However, the objective of our study is to investigate situations in which tablebased approaches are not guaranteed to succeed. In the remainder of this section, we specify the aspects of our learning problem that, in ways similar to real-world problems, render table-based approaches infeasible.

\subsection{Partial observability}

In an MDP, the current system state and action completely determine the dynamics of the ensuing transition. However, in a number of RL applications, perceptual aliasing (Whitehead and Ballard 1991) and noisy sensors (Stone et al. 2005) deny an agent direct access to the underlying system state. In principle the agent can keep a record of its past observations, and effectively use this memory as a means to reconstruct the system state (Lin and Mitchell 1993; McCallum 1996). Indeed the seminal work of Åström (1965) demonstrates that by keeping a "belief state" that is updated based on incoming observations, an agent can eventually disambiguate states perfectly. However, the complexity of doing so is forbidding even in the context of planning (with known transition dynamics) (Cassandra et al. 1994), and is yet to scale to large problems (Pineau et al. 2006). Using experience to disambiguate states in partially observable environments is typically feasible only in very small problems (Chrisman 1992; McCallum 1995; Bakker et al. 2003). In effect, learning agents in most RL applications have to treat "observed states" as states, and their performance varies depending on the validity of this assumption (Nevmyvaka et al. 2006).

Each cell in our task MDP corresponds to a state. In order to model partial observability, we constrain the learner to use an observed state $o$, which, in general, can be different from the true state $s$. Our scheme to pick $o$ based on $s$ is depicted in Fig. 2. Given $s$, we consider all the cells that lie within $d_{x}$ from it along the $x$ direction and within $d_{y}$ along the $y$ direction: from among these cells, we pick one uniformly at random to serve as the corresponding observed state $o$. Controlling $d_{x}$ and $d_{y}$ allows us to vary the extent of partial observability.

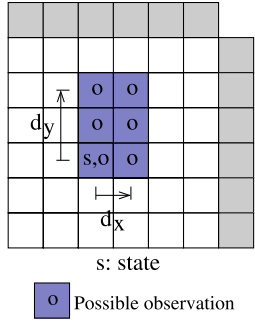

(a)

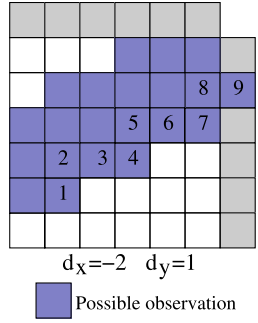

(b)

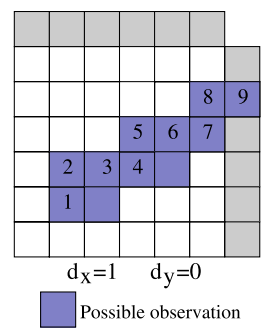

(c)

Fig. 2 An implementation of partial observability in the example MDP from Fig. 1. (a) Variables $d_{x}$ and $d_{y}$ (themselves generated randomly based on parameter $\sigma$ ) define a rectangle with the true state at a corner; cells within this rectangle are picked uniformly at random to constitute observed states. (b) A trajectory of true states 1 through 9 , and the set of all possible observed states that could be encountered during this trajectory when $d_{x}=-2$ and $d_{y}=1$. (c) For the same trajectory, the set of possible observed states when $d_{x}=1$ and $d_{y}=0$ 
Before starting a learning run, we fix $d_{x}$ and $d_{y}$ : each is sampled from a Gaussian distribution with zero mean and a standard deviation equal to $\sigma$, and then rounded to the nearest integer. Note that $d_{x}$ and $d_{y}$ can be positive, negative, or zero. Figures 2(b) and 2(c) show an illustrative trajectory of states numbered 1 through 9. Under different settings of $d_{x}$ and $d_{y}$, the figures show the set of all possible observed states that could result while the agent traces its trajectory. As is apparent from the figures, by keeping $d_{x}$ or $d_{y}$ fixed for the entire course of a learning run (i.e., by not changing them from episode to episode), the state noise encountered by the agent during its lifetime is systematic in nature. Informal experimentation with a number of schemes for implementing state noise suggests that biased noise tends to affect learning more severely than zero-mean noise. The magnitude of the noise, implemented through $d_{x}$ and $d_{y}$, is controlled by the single free parameter $\sigma$, which we vary in our experiments. Setting $\sigma$ to 0 ensures complete observability of state. Progressively larger values of $\sigma$ lead to observed states that are farther apart from the agent's true state, and render the agent's interaction with the environment non-Markovian.

\subsection{Function approximation}

The function approximation scheme in our learning problem is motivated by "CMAC" (Albus 1981), a popular method that is used in a number of RL applications (Singh and Sutton 1996; Stone et al. 2005; İpek et al. 2008). At each decision making step, we provide the learning agent a vector of $n_{f}$ features to describe its observed state. Each feature is a square "tile", with a binary activation: 1 within the boundary of the tile and 0 outside. Tiles have a fixed width $w$, which serves as a parameter in our experiments that determines the extent of generalization between states while learning. The centers of the tiles are chosen uniformly at random among non-terminal cells in the MDP. Figure 3 continues the example from Fig. 1, describing the architecture used for function approximation. In Fig. 3(a), nine tiles (numbered 1 through 9) are used by the function approximator. The tile width $w$ is set to 3; for illustration, four among the nine tiles are shown outlined.

Notice that every non-terminal cell in Fig. 3(a) is covered by at least one tile: i.e., every cell has at least one feature that is active. Indeed we ensure that complete coverage is always achieved, in order that non-trivial decisions can be made at every cell. Clearly, not

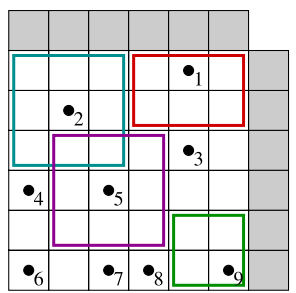

Centers of RBFs $\left(n_{f}=9\right)$

(a)

\begin{tabular}{c|c|c|c} 
& $\mathbf{N}$ & $\mathbf{E}$ & \\
\hline $\mathrm{f}_{1}$ & 2 & -1 \\
$\mathrm{f}_{2}$ & -8 & 3 \\
$\mathrm{f}_{3}$ & 0 & 6 & \\
$\mathrm{f}_{4}$ & 5 & 5 \\
$\mathrm{f}_{5}$ & 4 & -6 & \\
$\mathrm{f}_{6}$ & -3 & 2 \\
$\mathrm{f}_{7}$ & 2 & 3 \\
$\mathrm{f}_{8}$ & 4 & 1 & \\
$\mathrm{f}_{9}$ & -2 & 7 &
\end{tabular}

(b)

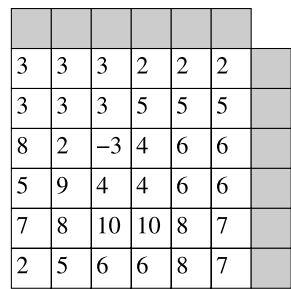

Larger of activation values

(c)

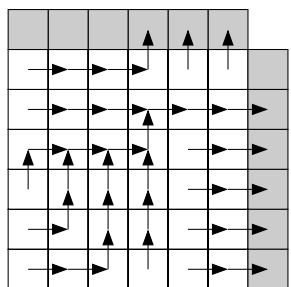

Greedy policy

(d)

Fig. 3 Function approximation in example MDP from Fig. 1. (a) A randomly chosen subset of cells (numbered 1 through 9) are the centers of overlapping tiles (giving $\chi=\frac{9}{36}=0.25$ ). The tile width $w$ is set to 3 ; tiles 1, 2, 5, and 9 are shown outlined (and clipped at the boundaries of the non-terminal region). (b) Table showing coefficients associated with each tile for actions $\mathbf{N}$ and $\mathbf{E}$. (c) The activation value of each cell for an action is the sum of the weights of the tiles to which it belongs. The figure shows the higher activation value (among $\mathbf{N}$ and $\mathbf{E}$ ) for each cell. (d) Arrows mark a policy that is greedy with respect to the activations: i.e., in each cell, the action with a higher activation value is chosen. In general the agent will take the greedy action from its observed state 
all the cells could be covered if the number of tiles $\left(n_{f}\right)$ and the width of each tile $(w)$ are both small; in all our experiments, we set these parameters such that in conjunction they can facilitate complete coverage of all non-terminal cells. The placement of the $n_{f}$ tiles is performed randomly, but preserving the constraint that all non-terminal cells be covered. In order to implement this constraint, we first place the tiles in regular positions that guarantee complete coverage, and then repeatedly shift tiles, one at a time, to random positions while still preserving complete coverage. Rather than treat $n_{f}$ directly as a parameter in our experiments, we normalize it by dividing by the number of non-terminal cells: $(s-1)^{2}$. The resulting quantity, $\chi=\frac{n_{f}}{(s-1)^{2}}$, lies in the interval $(0,1]$, and is more appropriate for comparisons across different problem sizes. In Fig. 3(a), $n_{f}=9$ and $s=7$, yielding $\chi=0.25$. We treat $\chi$ as a parameter in our experiments. As we shortly describe, $\chi$ determines the resolution with which independent actions can be taken from neighboring cells. In this sense, $\chi$ measures the "expressiveness" of the function approximation scheme.

Given the set of features for its observed state, the agent computes a separate linear combination for each action, yielding a scalar "activation" for that action. For illustration, consider Fig. 3(b), which shows a set of coefficients for each feature and action. It is these coefficients (or "weights") that the agent updates when it is learning. While learning, the agent may take any action from the states it visits. However, while evaluating learned behavior, we constrain the agent to take the action with the higher activation, breaking ties evenly. Figure 3(b) shows the higher of the resulting activations for the two possible actions at each cell in our illustrative example; Fig. 3(d) shows the action with the higher activation.

In effect, the only free parameters for the learning agent to update are the sets of coefficients corresponding to each action. By keeping other aspects of the representation-such as the features and policy-fixed, we facilitate a fair comparison between different learning methods. In general, value function-based methods such as Sarsa $(\lambda)$ seek to learn weights that approximate the action value function. We expect that setting $\sigma=0$ and $\chi=1$ would favor them, as the optimal action value function can then be represented. While this is so under any value of $w$, setting $w=1$ replicates the case of table-based learning with no generalization. Higher settings of $w$ enforce generalization. Increasing $\sigma$ or reducing $\chi$ would likely shift the balance in favor of policy search methods, under which activations of actions are merely treated as action preferences. As Baxter and Bartlett (2001) illustrate, even in simple 2-state MDPs, with function approximation, it is possible that the optimal action value function cannot be represented, even if an optimal policy can be represented.

In summary the design choices listed in this section are the end products of a process of trial and error directed towards constructing a suite of instances that allow us to study trends in learning algorithms, rather than constructing instances that are challenging in themselves. Table 2 summarizes the parameters used in our framework. Parameters $s, p, \sigma, \chi$, and $w$, along with a random seed, fix a learning problem for our experiments. By averaging over

Table 2 Summary of learning problem parameters. The last column shows the ranges over which each parameter is valid and meaningful to test

\begin{tabular}{llll}
\hline Parameter & Property of & Controls & Range \\
\hline$s$ & Task & Size of state space & $\{2,3, \ldots, \infty\}$ \\
$p$ & Task & Stochasticity in transitions & {$[0,0.5)$} \\
$\sigma$ & Agent/task interface & Partial observability & {$[0, \infty)$} \\
$\chi$ & Agent & Expressiveness of func. approx. & $(0,1]$ \\
$w$ & Agent & Generalization of func. approx. & $\{1,3, \ldots, 2 s-3\}$ \\
\hline
\end{tabular}


multiple runs with different random seeds, we estimate the mean performance achieved by learning methods as a function of $s, p, \sigma, \chi$, and $w$. Note that even if these parameters do not perfectly replicate an instance of any specific sequential decision making in practice, they are capable of being varied in a controlled manner to measure their effect on learning algorithms.

It must be noted that the parameterized learning problem described above is limited in several respects. While it enables the study of the most central problem parametersproblem size, stochasticity, partial observability and function approximation-it does not likewise isolate several other aspects influencing practical implementations of RL. Foremost is the question of exploration, which is not very crucial in our setup due to the occurrence of start states uniformly at random. The learning agent only has two actions; in practice large or continuous action spaces are quite common. Understanding the effect of other aspects, such as computational and memory constraints, the variation among action values from a state, different types of state noise, the sparsity and spread of the rewards, and the average episode length, would also be important for designing better algorithms in practice. We hope that the experimental methodology introduced in this article will aid future investigation on such subjects.

In the next section, we provide brief descriptions of the learning algorithms used in our experiments; in Sect. 4, the algorithms are compared at a number of different parameter settings drawn from the ranges provided in Table 2. Along with the parameterized learning problem itself, the results of these experiments are an important contribution of our article.

\section{Methods in study}

As noted earlier, we compare two contrasting classes of learning methods in our study: online value function-based (VF) methods, and policy search (PS) methods. With the aim of comparing these classes themselves, we first evaluate various methods within each class to pick a representative. In this section, we describe the learning methods thus considered, and describe relevant implementation-specific details. Experiments follow in Sect. 4.

\subsection{On-line value function-based (VF) methods}

We compare three learning methods from the VF class: Sarsa $(\lambda)$ (Rummery and Niranjan 1994; Rummery 1995), Q-learning $(\lambda)$ (Watkins 1989; Watkins and Dayan 1992; Rummery 1995; Peng and Williams 1996; Sutton and Barto 1998), and Expected Sarsa( $\lambda$ ) (abbreviated "ExpSarsa $(\lambda)$ ") (Rummery 1995; van Seijen et al. 2009). These methods are closely related: they all continually refine an approximation of the action value function, making a constanttime update every time a new state is encountered. Yet the methods are distinguished by subtle differences in their update rules. We include these methods in our study to examine how their differences affect learning under function approximation and partial observability: settings under which theoretical analysis is limited. We proceed to describe the methods themselves.

$\operatorname{Sarsa}(\lambda)$ is a model-free value function-based method, which makes on-line, on-policy, temporal difference (TD) learning updates. The learning agent maintains an estimate of an action value function, $Q$, which is updated as it encounters sequences of states $(s)$, actions (a) and rewards $(r)$. In particular assume that the agent encounters the following trajectory, in which suffixes index decision steps:

$$
s_{t}, a_{t}, r_{t+1}, s_{t+1}, a_{t+1}, r_{t+2}, s_{t+2}, a_{t+2}, r_{t+3}, \ldots
$$


The agent updates $Q\left(s_{t}, a_{t}\right)$ by computing a target, $Q_{\text {Target }}\left(s_{t}, a_{t}\right)$, and taking an incremental step towards it as follows:

$$
Q\left(s_{t}, a_{t}\right) \leftarrow\left(1-\alpha_{t}\right) Q\left(s_{t}, a_{t}\right)+\alpha_{t} Q_{\text {Target }}\left(s_{t}, a_{t}\right),
$$

where $\alpha_{t} \in(0,1]$ is the learning rate for the update. Recall that in our architecture, $Q$ is represented as a linear function approximator; hence, the learning update is implemented through gradient descent. Under Sarsa(0), the "fully bootstrapping" version of Sarsa, the target is computed as follows:

$$
Q_{\text {Target }}^{\text {Sarsa }(0)}\left(s_{t}, a_{t}\right)=r_{t+1}+\gamma Q\left(s_{t+1}, a_{t+1}\right),
$$

where $\gamma \in[0,1)$ is a discount factor. ${ }^{3}$ Note that the target does not count the actual rewards accrued beyond time step $t+1$; rather, the discounted sum of these "future" rewards is substituted with its current estimate: $Q\left(s_{t+1}, a_{t+1}\right)$. By contrast, a Monte Carlo method, Sarsa(1) computes its estimates wholly from sample returns, as:

$$
Q_{\text {Target }}^{\text {Sarsa }(1)}\left(s_{t}, a_{t}\right)=r_{t+1}+\gamma \sum_{k=1}^{\infty} \gamma^{k-1} r_{t+1+k} .
$$

This target would not change depending on the actual states that the trajectory visited, but only based on the sequence of rewards obtained. This makes Monte Carlo methods less dependent on the state signal than fully bootstrapping methods. Both methods still try to estimate state-action values, and therefore rely on being able to precisely detect $s_{t}$ and represent $Q_{\text {Target }}^{\text {Sars }}\left(s_{t}, a_{t}\right)$. In general, intermediate methods that implement varying extents of bootstrapping can be conceived by varying the "eligibility trace" parameter $\lambda \in[0,1]$. The estimated target for $Q\left(s_{t}, a_{t}\right)$ used by $\operatorname{Sarsa}(\lambda)$ is:

$$
Q_{\text {Target }}^{\operatorname{Sarsa}(\lambda)}\left(s_{t}, a_{t}\right)=r_{t}+\gamma\left\{(1-\lambda) Q\left(s_{t+1}, a_{t+1}\right)+\lambda Q_{\text {Target }}^{\mathrm{Sars}(\lambda)}\left(s_{t+1}, a_{t+1}\right)\right\} .
$$

For the case of discrete MDPs, in which $Q$ can be maintained as a table, Singh et al. (2000) show that by following a policy that is "greedy in the limit" with respect to $Q$, and which performs an infinite amount of exploration, Sarsa(0) will ultimately converge to the optimal action value function $Q^{*}$, from which the optimal policy $\pi^{*}$ can be derived by acting greedily. For linear function approximation schemes such as in our parameterized learning problem, Perkins and Precup (2003) show that convergence to a fixed point can be achieved by following a method similar to $\operatorname{Sarsa}(0)$.

We use a standard implementation of $\operatorname{Sarsa}(\lambda)$ with binary features, a linear representation, and replacing eligibility traces (see Sutton and Barto 1998, p. 212). While learning, the agent follows an $\epsilon$-greedy policy. We treat both the exploration strategy and the schedule for annealing the learning rate as parameterizable processes. We follow an $\epsilon_{u}$-greedy exploration policy during episode $u$, keeping $\epsilon_{0}$ as a free parameter, and $\epsilon_{U}=0.01$, where $U$ is the total number of training episodes. Intermediate values of $\epsilon_{u}$ are set based on a harmonic sequence going from $\epsilon_{0}$ to 0.01 . We use such a schedule based on empirical evidence of its effectiveness.

Interestingly, informal experimentation shows us that a similar annealing schedule is also the most effective for the learning rate $\alpha$; i.e., we keep $\alpha_{0}$ as a free parameter and anneal it

\footnotetext{
${ }^{3}$ It is legitimate to use $\gamma=1$ in episodic tasks. We do so in our experiments (see Sect. 4).
} 
harmonically to 0.01 at the end of training. Since features are binary, we divide the mass of each update equally among the features that are active under the state-action being updated. It is worth noting that theoretically-motivated update rules exist for annealing the learning rate. For example, Hutter and Legg (2008) derive a rule based on minimizing the squared loss between estimated and true values. However, their approach is only viable with tabular representations of $Q$, and further, only in continuing (rather than episodic) tasks.

Apart from $\lambda, \epsilon_{0}$, and $\alpha_{0}$, yet another parameter influencing Sarsa $(\lambda)$ is the setting of the initial weights (coefficients in the linear representation). In our experiments, we set all the weights initially to $\theta_{0}$, which is our final method-specific parameter. Table 3 summarizes the parameters defining $\operatorname{Sarsa}(\lambda)$. These parameters also apply to other methods in the VF class, which we now describe.

Whereas $\operatorname{Sarsa}(\lambda)$ computes its target for time $t$ based on the action to be taken at time $t+1-a_{t+1}-\operatorname{ExpSarsa}(\lambda)$ and Q-learning $(\lambda)$ compute their targets (and make learning updates) before $a_{t+1}$ is chosen. Once $s_{t+1}$ is reached, $\operatorname{ExpSarsa}(\lambda)$ computes its target based on an expectation over the possible choices of $a_{t+1}$ while following the current $\epsilon$-greedy policy $\pi_{t+1}$ :

$$
\begin{aligned}
Q_{\text {Target }}^{\text {Exparsa }(\lambda)}\left(s_{t}, a_{t}\right)= & r_{t}+\gamma\left\{(1-\lambda) Q\left(s_{t+1}, a_{t+1}\right)\right. \\
& \left.+\lambda \sum_{a \in A} \mathbb{P}\left\{a \mid s_{t+1}, \pi_{t+1}\right\} Q_{\text {Target }}^{\operatorname{ExpSarsa}(\lambda)}\left(s_{t+1}, a\right)\right\} .
\end{aligned}
$$

This alteration leads to a reduced variance in the update, as a sampled action value is now replaced with a smoothed-out estimate. It is shown by van Seijen et al. (2009) that like Sarsa(0), ExpSarsa(0) can also be made to converge to the optimal policy in discrete, finite MDPs. Q-learning $(\lambda)$ differs from Sarsa and ExpSarsa in that it is an off-policy method: rather than learning the action value function of the policy being followed, $\pi_{t}$, Q-learning $(\lambda)$ seeks to directly learn the action values of the optimal policy $\pi^{*}$. This objective is achieved by computing the target as follows:

$$
Q_{\text {Target }}^{Q \text {-learning }(\lambda)}\left(s_{t}, a_{t}\right)=r_{t}+\gamma\left\{(1-\lambda) \max _{a \in A} Q\left(s_{t+1}, a\right)+\lambda Q_{\text {Target }}^{Q \text {-learning }(\lambda)}\left(s_{t+1}, a_{t+1}\right)\right\} .
$$

Sutton and Barto (1998, see p. 184) refer to the update rule above as a "naïve" implementation of Q-learning with eligibility traces, because the rule lacks technical justification as a proper TD learning update. By contrast, there are some sound variations of Q-learning with eligibility traces (Watkins 1989; Peng and Williams 1996), under which updates additionally have to account for whether chosen actions were greedy or non-greedy. We refer the reader to the Ph.D. thesis of Rummery (1995, see Chap. 2) for an excellent presentation of various

Table 3 Summary of parameters used by methods within VF. The last column shows the ranges over which we tune each parameter

\begin{tabular}{lll}
\hline Parameter & Controls: & Range \\
\hline$\lambda$ & Eligibility traces & {$[0,1]$} \\
$\alpha_{0}$ & Initial learning rate & {$[0.1,1]$} \\
$\epsilon_{0}$ & Initial exploration rate & {$[0.1,1]$} \\
$\theta_{0}$ & Initial weights & {$[-10.0,10.0]$} \\
\hline
\end{tabular}


TD update rules. Note that Rummery refers to Sarsa as "modified Q-learning", and to Expected Sarsa as "summation Q-learning". It would exceed the scope of this article to undertake an extensive study comparing all possible variants of TD update rules. Rather, a novel contribution of our experiments is to consider three among them- $\operatorname{Sarsa}(\lambda), \operatorname{ExpSarsa}(\lambda)$, and (naïve) Q-learning $(\lambda)$ - in the presence of function approximation and partial observability. Indeed our results show that under this setting, hitherto uncharacterized patterns in performance emerge.

As with $\operatorname{Sarsa}(\lambda)$, we parameterize $\operatorname{ExpSarsa}(\lambda)$ and Q-learning $(\lambda)$ to control their learning and exploration rates, as well as their initial weights. The corresponding parameters, $\alpha_{0}, \epsilon_{0}$ and $\theta_{0}$, are summarized in Table 3 . Henceforward we drop the " $\lambda$ " from $\operatorname{Sarsa}(\lambda)$, $\operatorname{ExpSarsa}(\lambda)$, and Q-learning $(\lambda)$, and refer to these methods simply as Sarsa, ExpSarsa, and Q-learning, respectively. We do so to highlight that these methods are no longer only parameterized by $\lambda$ in our experiments - so are they by $\alpha_{0}, \epsilon_{0}$, and $\theta_{0}$.

Note that setting $w>1$ in our parameterized learning problem introduces generalization, and further, setting $\chi<1$ reduces the expressiveness of the function approximator. Thus, in general, the approximate architectures used are incapable of representing the optimal action value function $Q^{*}$. Even with full expressiveness $(\chi=1)$, if using generalization $(w>$ 1 ), methods from VF are not guaranteed to converge to the optimal action value function. And even if these methods approximate the action value function well, as defined through the Bellman error, greedy action selection might yet pick suboptimal actions in regions of inaccurate approximation, resulting in low long-term returns (Kalyanakrishnan and Stone 2007).

A bulk of the research in RL with linear function approximation has been in the context of prediction: estimating the value function of a fixed policy (without policy improvement). An early result due to Sutton (1988) establishes that TD(0) with linear function approximation converges when the features used are linearly independent; Dayan and Sejnowski (1994) extend this result to $\operatorname{TD}(\lambda), \forall \lambda \in[0,1]$, while Tsitsiklis and Van Roy (1997) show convergence for the more realistic case of infinite state spaces and linearly dependent features. Although most results for the convergence of linear TD learning are for estimating values of the policy that is used to gather experiences, the more general (and potentially useful) case of off-policy learning has also been addressed (Precup et al. 2001; Sutton et al. 2009).

The problems in learning approximate value functions on-line primarily arise due to the nonstationarity and bias in the targets provided to the function approximator (Thrun and Schwartz 1993). The best theoretical guarantees for learning control policies with approximate schemes come with several restrictions. Most results are limited to linear function approximation schemes; in addition some make demands such as Lipschitz continuity of the policy being learned (Perkins and Precup 2003) and favorable initial conditions (Melo et al. 2008). Results tend to guarantee convergence of certain updating schemes, but invariably lack desirable guarantees about the long-term reward that will be accrued at convergence (Sabes 1993; Perkins and Pendrith 2002; Perkins and Precup 2003).

In recent work, Maei et al. (2010) introduce the Greedy-GQ algorithm, which provably converges while making off-policy learning updates to a linear function approximator. Unfortunately, Greedy-GQ requires that the policy followed while learning stay fixed, preventing the agent from actively exploring based on the experiences it gathers. Thus, $\epsilon$-greedy exploration, with $\epsilon<1$, violates the assumptions needed for Greedy-GQ to converge; our informal experiments confirm that such a version of Greedy-GQ does not perform on par with the other methods we consider within the VF class. Thus, we do not include GreedyGQ in our extensive comparisons. 


\subsection{Policy search (PS) methods}

We include three methods from the PS class in our study: the Cross-entropy method (CEM) (de Boer et al. 2005), the Covariance Matrix Adaptation Evolution Strategy (CMAES) (Hansen 2009), and a genetic algorithm (GA). In addition we implement random weight guessing (RWG) to compare as a baseline.

CEM is a general optimization algorithm that has been used effectively as a policy search method on RL problems (Szita and Lőrincz 2006). In our linear representation, the vector of weights constitute the policy parameters to be adapted. The objective function, or "fitness" function, to be maximized is the expected long-term reward accrued by following the greedy policy that is derived from the weights. An iterative algorithm, CEM maintains and updates a parameterized distribution over the multi-dimensional search space. On each iteration, a population of \#pop points is sampled from the current distribution. Each point is evaluated, and the $\mu$ points with the highest fitness values are used to determine the distribution parameters for the next iteration. The update rule is such that with time the variance of the distribution shrinks and its mean gravitates towards regions of the parameter space with high fitness values. As is a common choice, in our experiments, we use a Gaussian distribution to generate sample points. We initialize the mean of this distribution to be the zero vector; along each dimension the variance is set to 1 (with no covariance terms). The update rule for Gaussian distributions is such that at every iteration, the updated distribution has as its mean and variance the sample mean and variance of the $\mu$ selected points (independently for each parameter). In general the update can also depend on the current distribution's mean and variance; further, noise can be added to the variance at each iteration to prevent premature convergence (Szita and Lőrincz 2006). We do not implement these variations in our experiments as they do not appear to have a significant effect in our domain.

Like CEM, the CMA-ES method also employs the principle of updating a distribution at each generation to maximize the likelihood of the $\mu$ points with the highest fitness values being generated. However, unlike CEM, CMA-ES tracks covariances across dimensions and actively monitors the search path in the parameter space leading up to the current generation. Handling several aspects in the search procedure, CMA-ES is a fairly sophisticated optimization technique; we refer the reader to descriptions from Hansen (2009) and Suttorp et al. (2009) for details. Nevertheless, we find it surprisingly straightforward to implement the algorithm based on existing code, which automatically sets most of the method-specific parameters. ${ }^{4}$ We set the initial distribution identically to the one set under CEM.

We implement GA in a manner akin to CEM and CMA-ES. On each generation, we spawn and evaluate \# pop policies; of these, the $\mu$ with the highest fitness values are selected to generate the next population. Specifically, pairs are chosen uniformly at random from the selected $\mu$ and crossed over to produce two offspring each. Policies are real-valued vectors over the space of parameters searched. Each parameter, restricted to the interval $[-1,1]$, is represented using a 32-bit Gray-coded string. To implement crossover between two individuals, the bit strings corresponding to each parameter are cut at a random location and matched across individuals, thereby yielding two offspring. To implement mutation, individuals are picked from the population with a small probability (0.05), and once picked, have each bit flipped with a small probability (0.1). Both under CEM and GA, we set $\mu$, the number of policies selected every generation to seed the next, to $15 \%$ of the population size \# pop. Experiments suggest that these methods are not very sensitive to $\mu$ values in

\footnotetext{
${ }^{4}$ See: http://www.lri.fr/ hansen/cmaes_inmatlab.html.
} 
this vicinity. CMA-ES uses a default value for $\mu$ depending on \#pop and the number of parameters searched.

In general, PS methods can work with a variety of representations. An illustrative example is the PS framework implemented by Kohl and Stone (2004) to optimize the forward walking speed of an Aibo robot. The gait they design has parameters describing trajectory positions and timings, which are combined using manually designed sets of rules. Evolutionary computation has been a particularly popular choice for PS; in particular several neuroevolutionary techniques have been tested on control tasks (Gomez and Miikkulainen 1999; Stanley 2004; Metzen et al. 2008). Typically the policy is represented using a neural network, whose topology and weights are evolved to yield policies with higher values.

In order to maintain a fair comparison with the VF methods in this study, we enforce that the methods chosen from PS employ the same representation, under which real-valued parameters are to be optimized (Sect. 2.3). In principle numerous evolutionary and optimization techniques apply to this problem: among others, amoeba, particle swarm optimization, hill climbing, and several variants of genetic and "estimation of distribution" algorithms. The reason we choose CEM and CMA-ES in our comparison is due to the several successes they have achieved in recent times (Szita and Lórincz 2006; Szita and Lórincz 2007; Hansen et al. 2009), which partly owes to their mathematically-principled derivation. We implement GA on the grounds that although it optimizes exactly the same parameters, it employs a bit string-based internal representation during its search, and thus is qualitatively different. Note that all the methods described above only use the ranks among fitness values in a generation to determine the next. In this manner, these methods differ from canonical policy gradient methods for RL (Sutton et al. 2000; Baxter and Bartlett 2001; Kakade 2001), which rely on the of gradients with respect to the policy parameters to identify a direction in the parameter space for policy improvement. Our policy is not analytically differentiable since it is deterministic.

The three PS methods described above take two parameters, listed in Table 4. Since fitness is defined as the expected long-term reward accrued by a policy, we estimate it by averaging the returns from \#trials episodes. The other method-specific parameter, \#gens, is the number of generations undertaken during the learning period. As a consequence, note that if the total number of training episodes is $U$, the population size in each generation is given by $\frac{U}{\# \text { trials } \times \text { gens }}$. Under RWG, we repeatedly generate policies, evaluate each for \#trials episodes, and retain the policy with the highest fitness. Informal experimentation shows that it is more effective to sample policies based on a Gaussian distribution for each parameter, rather than a uniform distribution.

\section{Experiments and results}

In this section, we present experimental results. First, in Sect. 4.1, we describe our experimental methodology. In Sect. 4.2, we perform comparisons within the VF and PS classes to

Table 4 Summary of parameters used by methods from PS. The last column shows the ranges over which we tune each parameter. The range shown for \#trials is used when the total number of episodes is 50,000, as in a majority of our experiments (see Sect. 4). The range is scaled proportionately with the total number of training episodes

\begin{tabular}{lll}
\hline Parameter & Controls: & Range \\
\hline \#trials & Samples per fitness evaluation & {$[25,250]$} \\
\#gens & Generations & {$[5,50]$} \\
\hline
\end{tabular}


pick representative methods from each. These representative methods are further compared across a series of experiments in Sects. 4.3 through 4.7 to ascertain their interactions with parameters of the learning problem.

\subsection{Experimental methodology}

As defined in Sect. 2, a learning problem is fixed by setting $s, p, \sigma, \chi, w$, and a random seed. Additionally, before conducting an experiment, we fix $U$, the total number of learning episodes conducted. Recall from Tables 3 and 4 that learning methods themselves have parameters: $\lambda, \alpha_{0}, \epsilon_{0}, \theta_{0}$, \#trials, and \#gens. In some experiments, we study the learning performance at fixed values of these method-specific parameters. However, note that even for a fixed method (say Sarsa), its best performance at different problem settings will invariably be achieved under different settings of its method-specific parameters $\left(\lambda, \alpha_{0}, \epsilon_{0}, \theta_{0}\right)$. In response we devise an automatic search procedure over the method-specific parameter space (4-dimensional for Sarsa) to find a configuration yielding the highest learned performance for a given problem instance and number of training episodes.

The search procedure-described schematically in Fig. 4 for a 2-dimensional parameter space-involves evaluating a number of randomly generated points in the space and iteratively halving the search volume, always retaining the region with the highest performance density. The procedure is necessarily inexact due to stochasticity in evaluations, and since performance might not be "well-behaved" over the region searched. Yet in practice we find that with sufficient averaging (2000 points per generation) and enough splits (5 times the number of dimensions searched), the procedure yields fairly consistent results. We suffix the method-specific parameter configurations returned by the search " $*$ " to indicate that they have been optimized for some task setting and number of training episodes. Thus, Sarsa* refers to an instance of Sarsa identified through the search procedure, its parameters being $\lambda^{*}, \alpha_{0}^{*}, \epsilon_{0}^{*}$, and $\theta_{0}^{*}$. Under $\operatorname{Sarsa}(\lambda)^{*}, \lambda$ is fixed, and only $\alpha_{0}, \epsilon_{0}$, and $\theta_{0}$ are optimized.

For clarity, we enumerate here the sequence of steps undertaken in each of our experiments.

1. We fix learning problem parameters $s, p, \sigma, \chi$, and $w$.

2. We fix the total number of training episodes $U$.

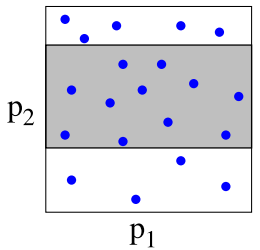

(a) Stage 1

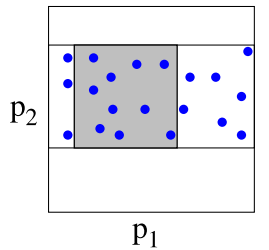

(b) Stage 2

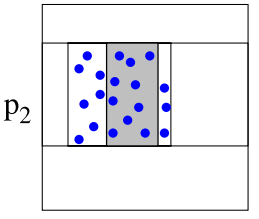

$\mathrm{p}_{1}$

(c) Stage 3

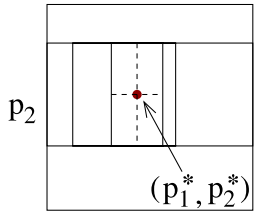

$\mathrm{p}_{1}$

(d) Final solution

Fig. 4 Illustration of a search over two parameters, $p_{1}$ and $p_{2}$. Initial ranges for each parameter are specified as inputs to the search. To begin, points are sampled uniformly from within the specified ranges. At each sampled point, a single learning run is conducted and its final performance recorded. Subsequently a split is performed to halve the search volume, retaining an axis-aligned region with the highest density of performance among all such regions. The process is repeated several times: with each split, attention is focused on a smaller part of the search space empirically found to contain the most successful learning runs. Note that at each stage, any parameter could lead to the best split $\left(p_{2}, p_{1}\right.$, and $p_{1}$ at stages $1,2,3$, respectively, in the illustration). At termination the midpoint of the surviving volume is returned 
3. Either we manually specify an instance of a learning method, or search for one, as described above, to maximize performance for the problem parameters and training episodes set in steps 1 and 2 .

4. With the chosen method instance, we conduct at least 1,000 independent trials of learning runs. Each trial is fixed by setting a different random seed, which can generate additional seeds for the learning problem (to determine features and rewards) and the learning method (to explore, sample, etc.).

5. Each learning trial above results in a fixed policy. We estimate the performance of this policy through 1,000 Monte Carlo samples. (Although sometimes a policy can be evaluated exactly through dynamic programming, the presence of function approximation and partial observability make it necessary to estimate performance through sampling.) Note that methods from VF and PS are both evaluated based on a greedy policy with respect to the learned weights.

6. Since all the rewards in our parameterized learning problem are non-negative, we find that problems with larger state spaces invariably lead to policies with higher absolute rewards. To facilitate meaningful comparison across problems with different parameter settings, we linearly scale the performance of a policy such that 0 corresponds to the value, under the same settings, of a random policy, and 1 to that of an optimal policy. In our graphs, we plot this normalized performance measure. Note that our careful design of the task MDP allows us to compute the performance values of random and optimal policies at each setting, even if the settings themselves preclude the learning of optimal behavior by an agent. Policies that are "worse than random" have normalized performance values less than zero.

7. We report the normalized performance achieved (over all trials), along with one standard error (typically these are small and sometimes difficult to distinguish visually in our graphs). Note that standard errors do not apply to the results of our parameter search, such as to find $\lambda^{*}$ under some problem instance. For any task instance, the methodspecific parameter search is conducted exactly once.

In summary: the steps outlined above aim to provide each method the best chance of success for a given problem instance and training time, and then to fairly evaluate and compare competing methods. Having specified our methodology, we proceed to describe results from our experiments.

\subsection{Picking representative methods and setting the training period}

The first phase in our experiments is to pick representative learning methods from the VF and PS classes. We now present comparisons among methods from these classes. We also describe how we set the number of training episodes for learning runs in our study.

\subsubsection{Picking a representative method from VF}

In comparing methods from the VF class, we observe that the method-specific parameter with the most dominant effect on performance is the setting of initial weights, $\theta_{0}$. For illustration consider Fig. 5. In the experiments reported therein, we compare $\operatorname{Sarsa}(0)$, ExpSarsa(0), and Q-learning $(0)$. For all these methods, we find that a broad range of the parameters $\alpha_{0}$ and $\epsilon_{0}$ yield policies with high performance; we manually pick favorable settings from among these ranges. Q-learning $(0)$ and Sarsa(0) use $\alpha_{0}=0.8, \epsilon_{0}=0.8$, while ExpSarsa(0) uses $\alpha_{0}=0.8, \epsilon_{0}=0.2$. The total number of training episodes $U$ is set to 50,000 . 
The three methods show qualitatively similar patterns in performance as $\theta_{0}$ is varied. In Fig. 5(a), we find that all of them achieve near-optimal behavior at large settings of $\theta_{0}$, directly reflecting the merits of optimistic initialization (Even-Dar and Mansour 2001). Action values tend to lie in the range $[0,20]$; correspondingly we notice that "pessimistic" initialization of weights to lower values leads to noticeable degradation in performance. Note that the settings in Fig. 5(a) correspond to a fully expressive tabular representation with no generalization. As we introduce generalization by increasing $w$ to 5 (Fig. 5(b)), we observe a significant change in trend: both very high and very low initial weights lead to a marked decrease in the final performance. This trend persists as the expressiveness $\chi$ is reduced (Fig. 5(c)).

In Figs. 5(b) and 5(c), it is apparent that ExpSarsa(0) falls below Sarsa(0) and Qlearning $(0)$ at most settings of $\theta_{0}$. We posit that since it performs a weighted average over all next state-action values, updates under $\operatorname{ExpSarsa(0)}$ are likely to propagate error from state-actions that are encountered less frequently. For a perfect tabular representation, such as in Fig. 5(a), van Seijen et al. (2009) prove that ExpSarsa(0) updates have the same bias, but a lower variance, compared to updates under $\operatorname{Sarsa}(0)$. However, our results appear to suggest that when generalization is present (as $w$ is increased), and learning starts with a stronger initial bias (by setting $\theta_{0}$ farther away from the true action values), ExpSarsa(0) suffers more from the error in its updates. Extending this argument, we could expect ExpSarsa to perform worse at high values of $\alpha_{0}$ and $\epsilon_{0}$ when generalization is used. Shortly we present the evidence for such a phenomenon.

We design three problem instances to further investigate differences between Sarsa, ExpSarsa, and Q-learning. Table 5 summarizes these problem instances. Instance $I_{1}$ corresponds to a fully-expressive tabular representation with no generalization, under which all three methods enjoy provable convergence guarantees. Expressiveness is reduced and generaliza-

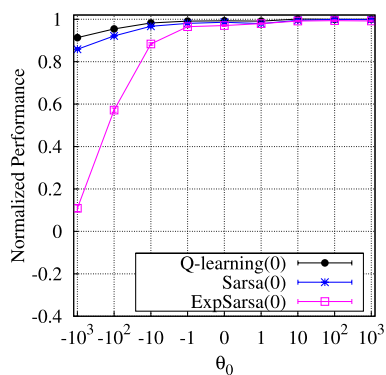

(a) $\chi=1, w=1$.

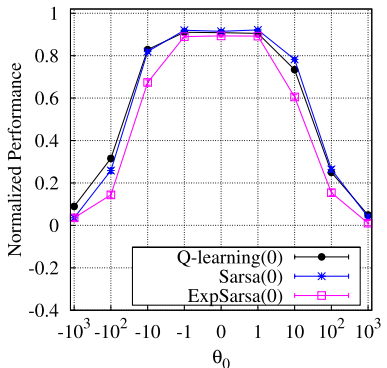

(b) $\chi=1, w=5$.

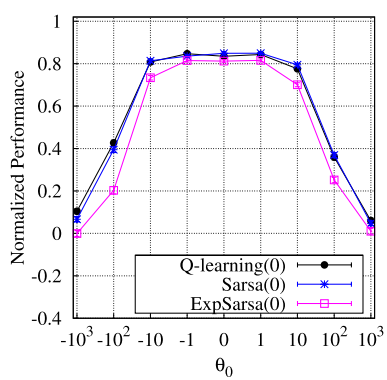

(c) $\chi=0.6, w=5$.

Fig. $5\left[s=10, p=0.2, \sigma=0\right.$.] Plots showing the effect of the initial weights $\theta_{0}$ on the performance of on-line value function-based methods. Note the irregular spacing of points on the $\mathrm{x}$ axis. Plot (a) corresponds to an exact tabular representation with no generalization. Generalization is introduced in (b) by increasing $w$; additionally the expressiveness $\chi$ is reduced in (c)

Table 5 Parameter settings for illustrative problem instances $I_{1}, I_{2}$, and $I_{3}$

\begin{tabular}{llllll}
\hline Problem instance & $s$ & $p$ & $\chi$ & $w$ & $\sigma$ \\
\hline$I_{1}$ & 10 & 0.2 & 1 & 1 & 0 \\
$I_{2}$ & 10 & 0.2 & 0.5 & 7 & 0 \\
$I_{3}$ & 10 & 0.2 & 1 & 1 & 4 \\
\hline
\end{tabular}


tion introduced in $I_{2}$. While $I_{1}$ and $I_{2}$ are both devoid of state noise, $I_{3}$ is identical to $I_{1}$ except for its higher setting of $\sigma$.

Figure 6 plots the performance of Sarsa*, ExpSarsa* and Q-learning* on $I_{1}, I_{2}$, and $I_{3}$. Notice that under $I_{1}$, all the methods achieve near-optimal behavior at the end of 50,000 episodes of training. While optimal behavior is not to be expected under $I_{2}$, it becomes apparent that ExpSarsa* trails the other methods in this problem ( $\mathrm{p}$-value $<10^{-4}$ ). This finding parallels the inference we draw from Fig. 5: generalization and function approximation adversely affect ExpSarsa, as its learning updates propagate more bias than either Sarsa or Q-learning.

Recall that $I_{3}$ is identical to $I_{1}$, except that it introduces state noise. Thus, when compared with $I_{1}$, we observe that all three methods suffer a significant drop in performance under $I_{3}$. Yet, the introduction of state noise does not appear to disadvantage any of the methods more than the others. Table 6 reports the optimized method-specific parameters found by our search strategy under the three problem instances. From the table, we see that the values of $\lambda^{*}$ found for all three methods under $I_{3}$ are significantly higher than the values found under $I_{1}$ and $I_{2}$. We may infer that reducing the reliance on bootstrapped estimates (by setting high values of $\lambda$ ) counteracts the error introduced in TD updates due to state noise. We also observe from Table 6 that the $\theta_{0}^{*}$ values found by our search strategy for each method and problem are as one may expect based on Fig. 5. These results affirm the reliability of our search strategy.

Predominantly we find that the VF methods compared above are not very sensitive to the learning rate parameter $\alpha_{0}$ and the exploration parameter $\epsilon_{0}$ within the ranges in which we optimize them: $[0.1,1]$ for both parameters. The only significant exception, to which we alluded earlier, is the case of ExpSarsa under $I_{2}$, which strongly favors lower $\alpha_{0}$ and

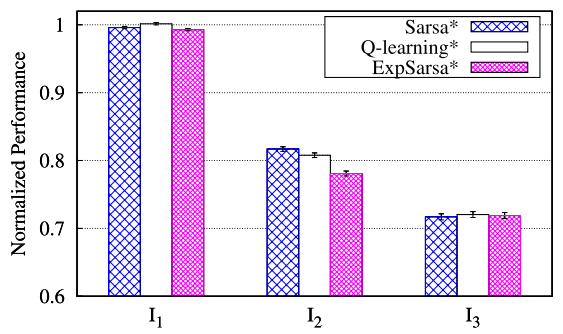

Fig. 6 Comparison of the performance of different VF methods on the three problem instances from Table 5. Under each instance, and for each of the methods-Sarsa, Q-learning, and ExpSarsa-a systematic search (see Sect. 4.1) identifies the method-specific parameter settings $\left(\alpha_{0}, \epsilon_{0}, \theta_{0}\right.$, and $\left.\lambda\right)$ yielding the highest performance after 50,000 episodes of training. The methods are marked " $*$ " as they are run under these optimized parameter settings

Table 6 For each of three methods-Sarsa, Q-learning, and ExpSarsa-the method-specific parameters yielding the highest performance (at 50,000 episodes of training), under problem instances $I_{1}, I_{2}$ and $I_{3}$. Figures are rounded to one place of decimal

\begin{tabular}{|c|c|c|c|c|c|c|c|c|c|c|c|c|}
\hline \multirow{2}{*}{$\begin{array}{l}\text { Problem } \\
\text { instance }\end{array}$} & \multicolumn{4}{|c|}{ Sarsa* } & \multicolumn{4}{|c|}{ Q-learning* } & \multicolumn{4}{|c|}{ ExpSarsa* } \\
\hline & $\bar{\lambda}$ & $\alpha_{0}$ & $\epsilon_{0}$ & $\theta_{0}$ & $\lambda$ & $\alpha_{0}$ & $\epsilon_{0}$ & $\theta_{0}$ & $\lambda$ & $\alpha_{0}$ & $\epsilon_{0}$ & $\theta_{0}$ \\
\hline$I_{1}$ & 0.3 & 0.6 & 0.6 & 7.0 & 0.2 & 0.3 & 0.7 & 8.7 & 0.2 & 0.5 & 0.7 & 6.7 \\
\hline$I_{2}$ & 0.1 & 0.7 & 0.7 & -1.0 & 0.2 & 0.7 & 0.8 & 0.8 & 0.1 & 0.9 & 0.2 & 0.8 \\
\hline$I_{3}$ & 0.9 & 0.5 & 0.5 & 8.9 & 0.9 & 0.2 & 0.9 & 6.4 & 0.8 & 0.6 & 0.8 & 6.7 \\
\hline
\end{tabular}


$\epsilon_{0}$ settings. For reference, we provide graphs plotting the performance of VF methods as a function of $\alpha_{0}$ and $\epsilon_{0}$ in Appendix A.

In summary: we find that Sarsa and Q-learning (albeit with a "naïve" implementation of eligibility traces) perform equally well on all our experiments; both methods outperform ExpSarsa on problems in which generalization is employed. We pick Sarsa as a representative method from the VF class for our subsequent experiments (Sects. 2.1 through 4.7).

\subsubsection{Picking a representative method from PS}

We reuse problem instances $I_{1}, I_{2}$, and $I_{3}$ to compare methods from the PS class. As noted in Sect. 3.2, two parameters have to be set for methods from this class: \#trials and \#gens. Optimizing over these parameters, we plot the performance of CEM*, CMA-ES*, GA* and RWG* $^{*}$ in Fig. 7. Unlike with the VF class, the ordering among the methods from PS stays consistent across the problem instances. In all cases, CEM* and CMA-ES* outperform GA* and $\mathrm{RWG}^{*}$ (p-value $<10^{-4}$ ). However, CEM* and CMA-ES* themselves register virtually identical performance: they cannot be separated with statistical significance on instances $I_{1}$ and $I_{3}$, although in $I_{2}$, CMA-ES* emerges the winner ( $p$-value $<0.02$ ).

It is worth noting that whereas all the VF methods in our study achieve their highest performance on instance $I_{1}$, all the methods from PS achieve theirs on $I_{2}$. 50,000 episodes is a relatively short duration of training for PS methods, which do not make effective use of individual transition samples, but rather, aggregate them in evaluating fitness. Greater generalization across the state space (as in $I_{2}$, where $w=5$ ) enables them to learn more quickly. In Sect. 4.6, we observe that if optimized for 500,000 episodes, PS methods do perform better at $w=1$.

The best parameter settings found for each PS method, under the three chosen problem instances, are listed in Table 7. Although we search over \#trials and \#gens, note that

Fig. 7 Comparison of the performance of different PS methods on the three problem instances from Table 5. Methods are marked " $*$ " to denote that method-specific parameters-\#trials and \#gens (except for RWG)-have been optimized for each task instance

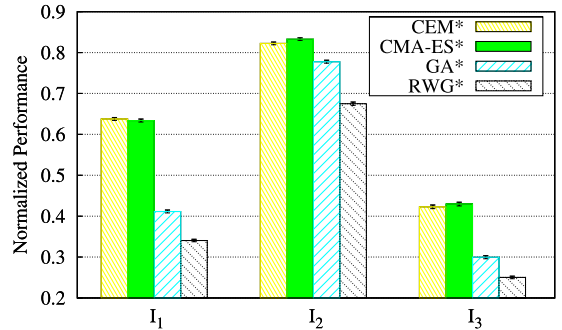

Table 7 For policy search methods, the method-specific parameters yielding the highest performance (at 50,000 episodes of training) for problem instances $I_{1}, I_{2}$, and $I_{3}$. Figures are rounded to the nearest integer. Under CEM, CMA-ES, and GA, the parameters searched are \#gens ("\#g") and \#trials ("\#t"). These parameters automatically fix the population size ("\# $p$ "), which is suffixed with "D" to denote that it is implicitly derived. Under RWG, only \#t is optimized; \# $g$ is implicitly derived. Derived parameters values are shown for reference

\begin{tabular}{|c|c|c|c|c|c|c|c|c|c|c|c|}
\hline \multirow{2}{*}{$\begin{array}{l}\text { Problem } \\
\text { instance }\end{array}$} & \multicolumn{3}{|c|}{ CEM $^{*}$} & \multicolumn{3}{|c|}{ CMA-ES* } & \multicolumn{3}{|c|}{$\mathrm{GA}^{*}$} & \multicolumn{2}{|c|}{ RWG* } \\
\hline & $\# g$ & $\# t$ & $\# p_{D}$ & $\# g$ & $\# t$ & $\# p_{D}$ & $\# g$ & $\# t$ & $\# p_{D}$ & $\# g_{D}$ & $\# t$ \\
\hline$I_{1}$ & 18 & 44 & 63 & 24 & 61 & 34 & 9 & 49 & 113 & 113 & 444 \\
\hline$I_{2}$ & 15 & 64 & 52 & 20 & 111 & 23 & 22 & 17 & 134 & 327 & 153 \\
\hline$I_{3}$ & 10 & 113 & 44 & 17 & 80 & 37 & 15 & 30 & 111 & 125 & 400 \\
\hline
\end{tabular}


thereby we implicitly set up a search over the population size \# pop used in every generation. This is a consequence of the relation that \#trials $\times \# g e n s \times \# p o p=U$, the total number of training episodes. From the table, we observe that CMA-ES* typically employs a smaller population size than $\mathrm{CEM}^{*}$, while $\mathrm{GA}^{*}$ maintains significantly larger population sizes.

Appendix B displays the performance of the various PS methods as a function of their input parameters. We observe a noticeable variance in the performance of all the methods over the parameter ranges considered. While CMA-ES* and $\mathrm{CEM}^{*}$ have comparable performance on all three problem instances, it is apparent that CMA-ES is more robust to parameter settings; i.e., it registers a higher performance over a wider range of settings. This makes CMA-ES overall a slightly more favorable candidate than CEM from the class of PS methods. Therefore, we select CMA-ES for our further experiments.

\subsubsection{Setting the training period}

Even if the problems in our experiments are themselves reasonably small, the extensive search and evaluation processes incur a significant amount of time during each experiment. One factor that plays a major role in determining the experimental running time is $U$, the total number of training episodes in each run. Setting $U=50,000$, as we have in the experiments reported thus far, it takes us roughly 1-2 hours to complete a single search and evaluation procedure, such as, for example, identifying Sarsa* and evaluating it under $I_{2}$. In this duration, we have roughly 200 processes running in parallel on a computing cluster with $2 \mathrm{GHz}$ CPUs. In general we do not find it feasible to conduct extensive experimentation under higher values of $U$ (although we do undertake such investigation under some interesting cases, such as in Sect. 4.6).

To gauge the implications of consistently setting $U=50,000$ in our subsequent comparisons, we run a single suite of experiments at multiple settings of $U$. Figure 8 shows the performance of Sarsa*, Q-learning*, ExpSarsa*, CEM*, and CMA-ES*; under problem instances $I_{1}, I_{2}$, and $I_{3}$; optimized for various settings of $U$. As expected we find that all the methods improve their performance with longer training periods. The gains from a longer training period are more marked among the methods from PS, as in general, methods from VF appear to plateau within a few thousands of episodes.

We observe from Fig. 8 that under all problem instances, the trend within methods in VF remains roughly the same at all values of $U$ : under $I_{1}$, the methods all achieve comparable performance; under $I_{2}$, ExpSarsa* performs poorest; and under $I_{3}$, Q-learning* . Likewise, no clear winner among $\mathrm{CEM}^{*}$ and CMA-ES* emerges in any of the instances, for any setting of $U$. Therefore, we may conclude that our choice of picking Sarsa* and CMA-ES* for further comparisons is justified. However, the choice of $U$ does affect comparisons between Sarsa* $^{*}$ and CMA-ES* themselves. Notice that up to 25,000 episodes, Sarsa* consistently outperforms CMA-ES*. Yet, from 50,000 episodes onward, CMA-ES* overtakes Sarsa* on

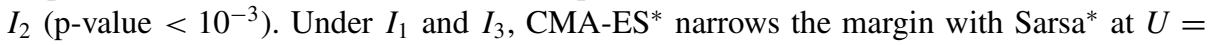
$1,000,000$, although it does not reach comparable performance.

The trends in Fig. 8 inform our interpretation of the results to follow in the remainder of this section. In general we expect that Sarsa will not significantly improve its performance beyond 50,000 episodes of training, whereas CMA-ES consistently improves at least up to 1000,000 episodes. Even so, in several instances, we find that CMA-ES outperforms Sarsa even at 50,000 episodes, validating this choice of $U$ as a meaningful comparison point between the methods.

In summary: our "within class" comparisons in VF and PS provide convincing evidence that Sarsa and CMA-ES are respectively the best methods to represent these classes in our 
Fig. 8 Plots showing the performance of different learning methods as the number of training episodes $U$ is varied. Each plot corresponds to a problem instance from Table 5. Note the irregular spacing of points on the $\mathrm{x}$ axis. At each point, the best performance achieved by three learning methods from VF (Sarsa*, Q-learning*, and ExpSarsa*) and two from PS (CEM* ${ }^{*}, \mathrm{CMA}^{\mathrm{E}}{ }^{*}$ ) is shown

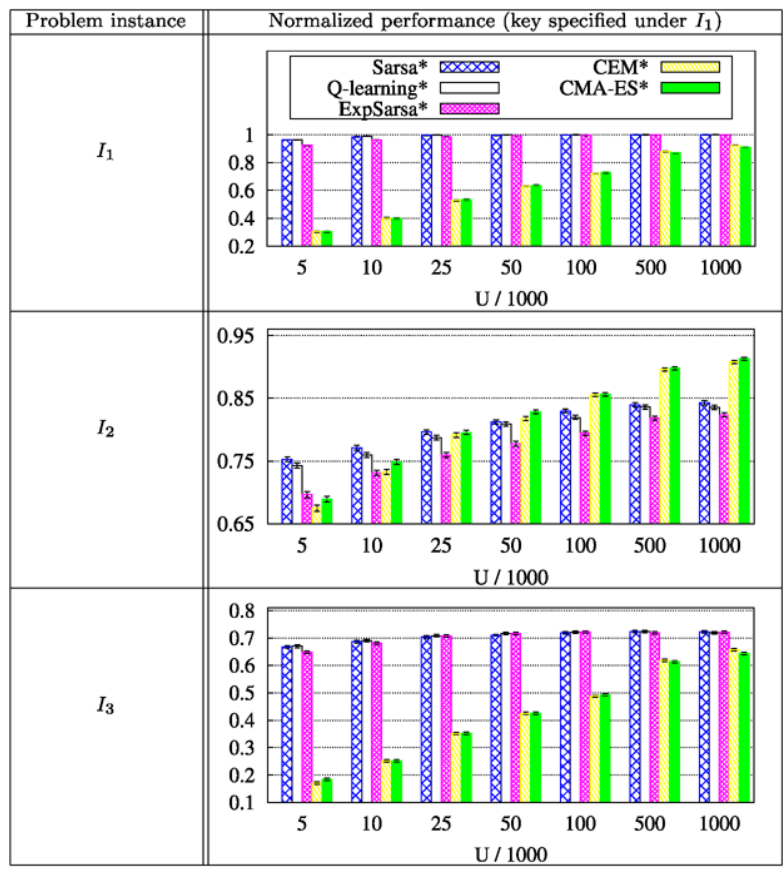

parameterized learning problem (except that Q-learning performs as well as Sarsa in VF). We now proceed to compare these methods as relevant problem parameters are varied. In each comparison (excepting cases in Sect. 4.6), the normalized performance of these methods after 50,000 episodes of training is considered while evaluating them.

\subsection{Problem size and stochasticity}

In our first set of "VF versus PS" experiments, we evaluate our learning methods as the size of the state space and the stochasticity of transitions in the task MDP are varied. Conjunctions of three settings of $s(6,10,14)$ and three settings of $p(0,0.2,0.4)$ are compared; results are plotted in Fig. 9. With complete expressiveness $(\chi=1)$, no generalization $(w=1)$, and full observability $(\sigma=0)$, all nine cases are akin to learning with a classical "tabular" representation.

The most striking observation from the plots in Fig. 9 is the disparity in the learning rates of Sarsa* and CMA-ES*. In all nine cases, Sarsa* reaches near-optimal behavior, and typically so within a few thousands of episodes. At 50,000 episodes of training, in none of the problems does CMA-ES* match the performance of Sarsa* (p-value $<10^{-4}$ ). The gap between the methods is to be expected, as by making learning updates based on every transition, VF methods make more efficient use of experience for learning than PS methods do. Note that Sarsa is still on-line and model-free; we expect model-based methods (Sutton 1990) and batch methods (Lin 1992; Lagoudakis and Parr 2003) to further improve sample efficiency.

For both Sarsa* and CMA-ES*, we notice a decrease in performance as $s$ is increased. This decrease is more marked for CMA-ES* ${ }^{*}$ as the dimensionality of the parameter space it searches increases quadratically with $s$. The effect of the stochasticity parameter in widening the gap between Sarsa* and CMA-ES* is also significant. The error bars plotted in the graphs 


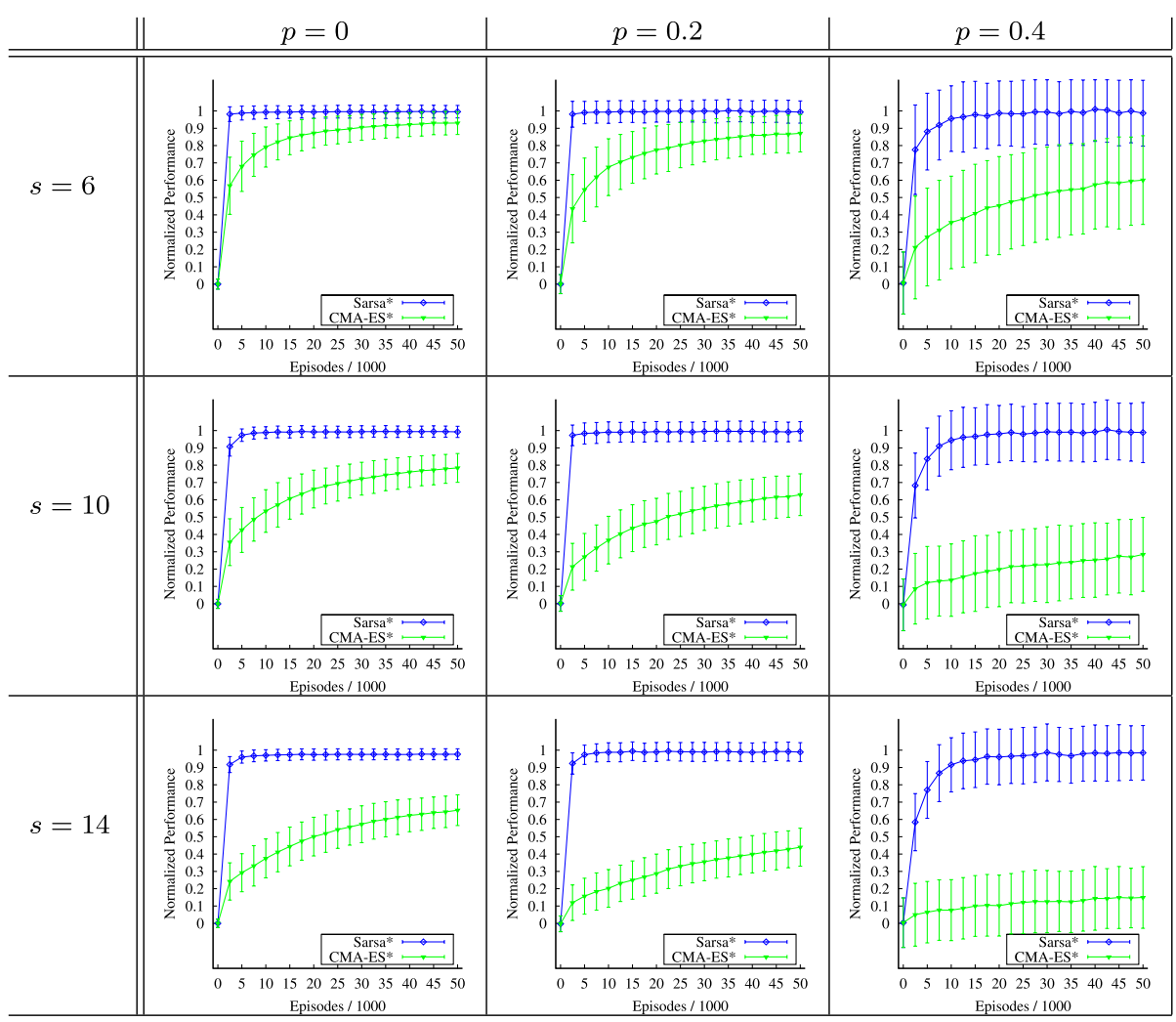

Fig. $9[\chi=1, w=1, \sigma=0$.] Sarsa* and CMA-ES* (optimized for 50,000 episodes of training) compared at different settings of $s$ and $p$. Unlike other plots in the article, in these learning curves, we plot one standard deviation in the performance (instead of one standard error)

show one standard deviation in performance (in all other graphs in the article, one standard error is shown). We observe that for both methods, the variance in performance increases as $p$ is increased, and further, that for any given problem, CMA-ES* displays a slightly higher variance than Sarsa*. As described in Sect. 2, note that even at $p=0$, there is stochasticity in the task, as the start state for each episode is picked uniformly at random.

Recall that the method-specific parameters of Sarsa* and CMA-ES* have been optimized for each problem and training period. While we do not note any significant patterns among the method-specific parameters thereby found under Sarsa*, we note that under CMA-ES*, \#trials ${ }^{*}$ gets consistently higher as $p$ is increased. For example, at $s=10$, the settings of \#trials* found by our search procedure are 38,77, and 152 for $p=0, p=0.2$, and $p=0.4$, respectively. In other words, CMA-ES* benefits from more evaluation trials in evaluating fitness values as the task stochasticity increases. Indeed recent research addresses the problem of tuning \#trials in an adaptive manner (Heidrich-Meisner and Igel 2009).

The primary inference from the set of experiments above is that Sarsa has significant advantages both in terms of the performance achieved and the variance in performance as problem size and stochasticity are increased. Not only is CMA-ES slower to learn, it demands better tuning of \#trials across different problem instances. To characterize the reasons underlying these observations, we turn to Cobb (1992), who separates the inductive biases in 
a reinforcement learner into "language" and "procedural" biases. The former corresponds to the representation used by the learner, which in this study, we have fixed to be the same for the methods compared. VF and PS methods are essentially separated by their procedural bias: how they updates weights in the representation. The language bias in the problem instances above- $\chi=1, w=1, \sigma=0$ - strongly favors the procedural bias of Sarsa. How would the methods fare if the language bias is changed? The experiments to follow examine the effects of state noise, generalization, and expressiveness.

\subsection{Partial observability}

In our second set of experiments, we study the effect of partial observability by increasing $\sigma$. We notice a conjunctive relationship between $\sigma$ and $w$, the generalization width. In response we conduct experiments with three settings of $\sigma(0,2,4)$ and three settings of $w(1,5,9)$. Results are plotted in Fig. 10: in each graph therein the performance of $\operatorname{Sarsa}(\lambda)^{*}$ is plotted at six values of $\lambda(0,0.2,0.4,0.6,0.8,1)$. The performance of CMA-ES* (which does not depend on $\lambda$ ) is also shown.

In general the best memoryless policies for Partially Observable MDPs (POMDPs) can be stochastic (Singh et al. 1994). Perkins and Pendrith (2002) show that in order to converge in POMDPs, it is necessary for methods such as Sarsa and Q-learning to follow policies that

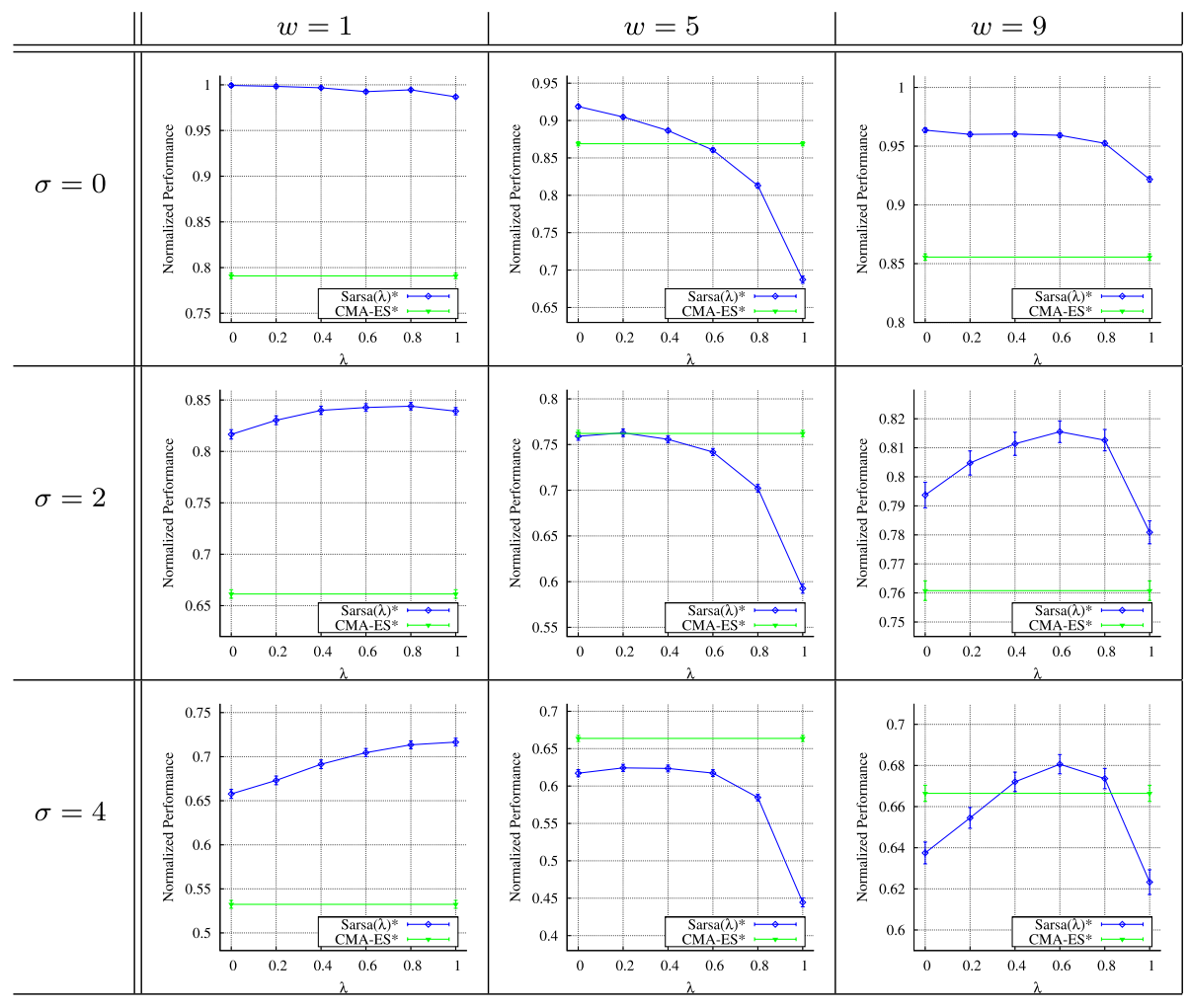

Fig. $10\left[s=10, p=0.2, \chi=1\right.$.] $\operatorname{Sarsa}(\lambda)^{*}$ and CMA-ES* compared at different settings of $\sigma$ and $w$. Under each plot, six regularly spaced values of $\lambda$ are chosen and the corresponding $\operatorname{Sarsa}(\lambda)^{*}$ evaluated. CMA-ES* appears as a line, as it does not depend on $\lambda$ 
are continuous in the action values, unlike the $\epsilon$-greedy policies used by the VF methods in our experiments. However, we do not observe any divergent behavior for $\operatorname{Sarsa}(\lambda)$ in the experiments reported here.

We notice that when $\sigma=0$ and $w=1$, the effect of $\lambda$ on the performance of $\operatorname{Sarsa}(\lambda)^{*}$ is not very pronounced. As soon as either $\sigma$ or $w$ is increased, intermediate values of $\lambda$ predominantly yield the highest performance for $\operatorname{Sarsa}(\lambda)^{*}$. These results echo the findings of Loch and Singh (1998), who demonstrate that deterministic policies learned using $\operatorname{Sarsa}(\lambda)$ with ample exploration perform quite well on a suite of benchmark POMDPs. Key to their success is the high values of $\lambda$ used (between 0.8 and 0.975 ), which weight true returns from actions much higher than estimated values.

As the generalization width $w$ is increased, notice that there is no longer a single winner between Sarsa* and CMA-ES*: VF methods no longer dominate PS methods when observability of state is limited. An intriguing trend that becomes apparent from Fig. 10 is that the performance of $\operatorname{Sarsa}(\lambda)^{*}$ is not monotonic with respect to $w$ : for most settings of $\sigma$ and $\lambda$, the highest performance is achieved at $w=1$, followed by $w=9$, with the lowest performance at $w=5$. In Sect. 4.6, we find further evidence of such anomalous patterns in the performance of Sarsa as $w$ is varied. Interestingly CMA-ES* registers its highest performance, for any fixed $\sigma$, at $w=5$ ( $w=9$ comes a close second). This trend arises as 50,000 episodes is a relatively short training duration for PS methods in this domain; generalization promotes quick initial learning. Experiments in Sect. 4.6 confirm that with 500,000 training episodes, CMA-ES performs best at $w=1$.

A recent variant of Sarsa $(\lambda)$ applied to POMDPs is SarsaLandmark (James and Singh 2009 ), in which $\lambda$ is set to 0 (full bootstrapping) when special "landmark" states (which are perfectly observable) are visited, but remains 1 at all other times (Monte Carlo). SarsaLandmark is not directly applicable in our domain as the agent receives no special information about landmark states. In recent work, Downey and Scanner (2010) propose a method to adaptively tune $\lambda$ while learning. Formally derived under a Bayesian framework, their algorithm-Temporal Difference Bayesian Model Averaging (TD-BMA)—is shown to outperform $\operatorname{Sarsa}(\lambda)$ for any fixed value of $\lambda$ on illustrative grid-world tasks. Our results highlight that tuning $\lambda$ is of particular relevance in problems with state noise and generalization; our parameterized learning problem therefore becomes an ideal testbed for evaluating adaptive approaches.

In Table 8 , we report the best initial weights, $\theta_{0}^{*}$, found for $\operatorname{Sarsa}(\lambda)^{*}$, under various settings of $\lambda, \sigma$, and $w$. The most noticeable pattern from the table is the favor for lower settings of $\theta_{0}$ as $w$ is increased. The best initial weights do not appear to change much as state noise and eligibility traces are varied.

Table $8[s=10, p=0.2, \chi=1.] \theta_{0}^{*}$ (initial weights under $\left.\operatorname{Sarsa}(\lambda)^{*}\right)$ for different problem instances. Each cell in the table corresponds to a setting of $\sigma, w$ (problem parameters), and $\lambda$ (Sarsa parameter); entries correspond to the value of $\theta_{0}$ found by searching for $\operatorname{Sarsa}(\lambda)^{*}$. Note that each search is only performed once

\begin{tabular}{|c|c|c|c|c|c|c|c|c|c|}
\hline \multirow[t]{2}{*}{$w$} & \multicolumn{3}{|l|}{$\lambda=0$} & \multicolumn{3}{|c|}{$\lambda=0.4$} & \multicolumn{3}{|c|}{$\lambda=1.0$} \\
\hline & $\sigma=0$ & $\sigma=2$ & $\sigma=4$ & $\sigma=0$ & $\sigma=2$ & $\sigma=4$ & $\sigma=0$ & $\sigma=2$ & $\sigma=4$ \\
\hline 1 & 8.7 & 7.4 & 5.0 & 8.0 & 7.9 & 7.8 & 7.8 & 7.5 & 9.6 \\
\hline 5 & 0.5 & 0.0 & -0.7 & 0.3 & 0.6 & 1.8 & 2.1 & 1.4 & 1.6 \\
\hline 9 & -0.3 & -0.3 & 0.6 & 0.1 & 0.0 & -0.6 & 0.1 & 0.5 & -0.8 \\
\hline
\end{tabular}




\subsection{Expressiveness of function approximator}

Continuing our study, we conduct experiments to gauge the role of the expressiveness parameter $\chi$ in determining the performance of learning methods. Again, we find no single winner among Sarsa* and CMA-ES* as $\chi$ is varied. The results shown in Fig. 11 are under fixed settings: $\sigma=0$ and $w=5$. The qualitative nature of the results does not change as $\sigma$ and $w$ are varied.

In the learning curve in Fig. 11(a), under $\chi=1$ (which allows the optimal action value function to be represented), Sarsa* displays quick learning to reach a normalized performance close to 0.9, whereas CMA-ES* fails to achieve comparable performance after 50,000 episodes. By contrast, at $\chi=0.4$ (Fig. 11(b)), we notice that Sarsa* suffers a dramatic drop in performance, plateauing at a normalized performance value close to 0.7 . At the same setting of $\chi$, CMA-ES* overtakes the learning curve of Sarsa* and reaches a significantly higher performance at 50,000 episodes ( $\mathrm{p}$-value $<10^{-4}$ ).

As $\chi$ is decreased, the representation for the value function and policy becomes increasingly handicapped. In Fig. 11(c), we observe that both under Sarsa and CMA-ES, performance decreases monotonically as $\chi$ is reduced. However, of the two methods, Sarsa suffers the more significant drop in performance as $\chi$ is reduced. Whereas Sarsa outperforms CMAES for $\chi \geq 0.7$ ( $\mathrm{p}$-value $<10^{-4}$ ), the opposite is true when $\chi \leq 0.5$ (p-value $<10^{-4}$ ). We do not observe any striking trends in the method-specific parameters of Sarsa* and CMA-ES* as $\chi$ is varied.

To the best of our knowledge, prior literature does not compare methods from VF and PS while constraining them to use the same representation. Our finding that CMA-ES is able to achieve good performance even under a representation that is extremely impoverished for approximating the value function suggests that it is a promising candidate in a large number of real-world domains in which feature engineering and representations are deficient. We posit that like the example constructed by Baxter and Bartlett (2001), many of the cases with $\chi<1$ allow for the representation of high-reward policies, but only admit poor approximations of the action value function. Notice that we do not have any irrelevant features in our learning problem: in the future it would be useful to incorporate such a setting, which is often encountered in practice. Non-linear function approximation would be an equally important avenue to explore.

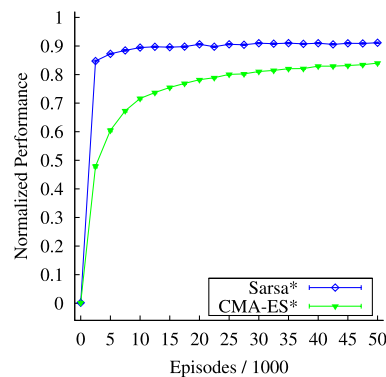

(a) $\chi=1$

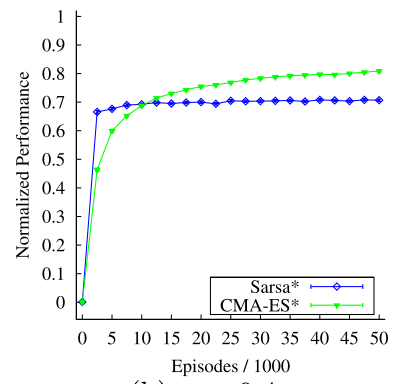

(b) $\chi=0.4$

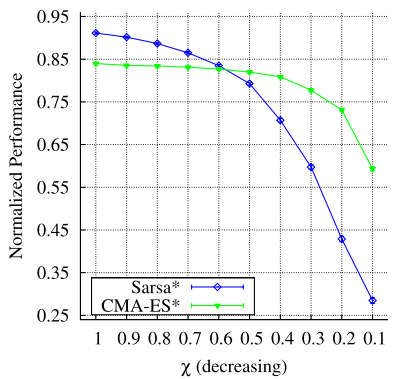

(c) Range of $\chi$

Fig. $11\left[s=10, p=0.2, w=5, \sigma=0\right.$.] Plots (a) and (b) show learning curves of Sarsa $(\lambda)^{*}$ and CMA-ES* at different values of $\chi$. Plot (c) shows the performance achieved after 50,000 episodes of training at different values of $\chi$ 


\subsection{Generalization of function approximator}

In Sect. 2.2, we noted that the generalization parameter $w$ plays a role in determining the relative order between Sarsa and CMA-ES at different values of $\sigma$. In examining the effect of $w$ more closely, we notice that although its effect on CMA-ES is fairly regular, its interaction with Sarsa is less predictable. Figure 12 shows the normalized performance of these methods at settings of $w$ varying from 1 (no generalization) to 15 (very broad generalization). In Fig. 12(a), the total number of training episodes is set to 50,000, while in Fig. 12(b), it is set to 500,000 .

We observe that with 50,000 training episodes, CMA-ES* performs its best at $w=3$, but with 500,000 episodes, its best performance is at $w=1$. We ascribe this effect to the benefit of generalizing early during the search, which quickly identifies the most promising actions in localized regions of the state space. With more time it becomes important to further discriminate among individual states, which it is most possible with $w=1$. It is not surprising that for both methods, the performance begins to drop sharply for $w>9$. Since in this problem, $s=10$, some non-terminal cells in the task MDP necessarily get activated by all the tiles present if the tile width exceeds 9. Indeed beyond $w=19$ (not shown in figure), no two cells in the MDP remain distinguishable.

Interestingly Sarsa* presents a less regular pattern in performance as $w$ is varied, as evinced by Fig. 12(a). We find that Sarsa* is most effective at $w=1$, but its performance suffers a dip until $w=5$; again a rise until $w=9$; before monotonically decreasing again. 50,000 episodes is a fairly long duration by VF standards, as apparent from several learning curves shown in the article (for instance, see Fig. 11(a)). It is clear that the irregular performance pattern of Sarsa* is not an artefact of training time, as the pattern essentially persists at 500,000 episodes of training (Fig. 12(b)). Also, notice the small error bars in both the plots: the pattern is systematic.

We investigate whether the irregular pattern in the performance of Sarsa persists as the problem size is increased beyond $s=10$. Figure 13 (see top row) indeed affirms that at $s=14$ and $s=18$, too, multiple local minima emerge in the performance as $w$ is varied. Curiously, under all three settings of $s$, we observe that as $w$ is varied, a correlation existsup to $w<s$-between the performance of Sarsa* and $\lambda^{*}$, the eligibility trace parameter optimized for each problem setting. The bottom row in Fig. 13 shows the values of $\lambda^{*}$ under each setting. Observe that for $w<s$ the local maxima and minima in $\lambda^{*}$ predominantly coincide with those of the normalized performance (recall that for $w \geq s$, states necessarily become aliased).

At present we do not have a conclusive explanation for the phenomenon described above. Since CMA-ES shows predictable variation with $w$, we surmise that the variation shown by Sarsa ultimately arises from its on-line updates to the value function. We speculate that

Fig. $12[s=10, p=0.2, \chi=1$, $\sigma=2$.] Performance of Sarsa* and CMA-ES* at different values of $w$, optimized in plot (a) for 50,000 training episodes, and in plot (b) for 500,000 training episodes

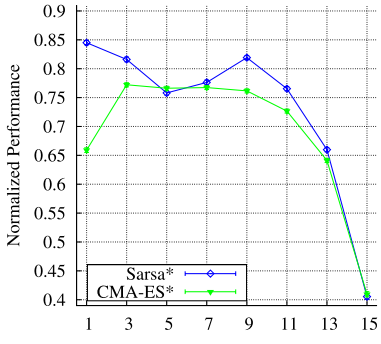

(a) episodes $\stackrel{\mathrm{w}}{=} 50,000$

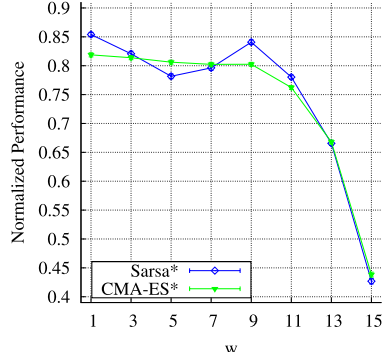

(b) episodes $\stackrel{\text { w }}{=} 500,000$ 


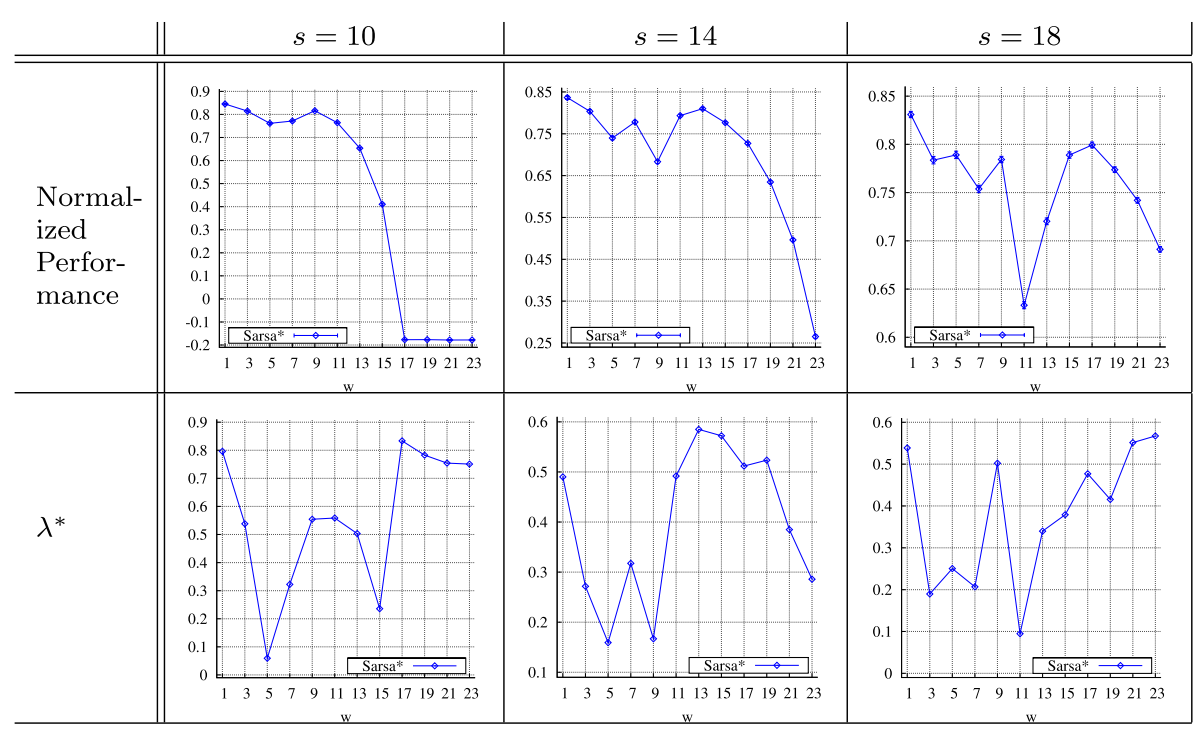

Fig. $13[p=0.2, \chi=1, \sigma=2$.] Analysis of Sarsa* as $s$ (columns) and $w$ (x axis in each plot) are varied. The top row shows the normalized performance achieved at each setting; correspondingly the bottom row shows $\lambda^{*}$ - the value of $\lambda$ found by searching for Sarsa* — for the same settings

"edge effects" in our tiling scheme, whereby states on the periphery of the grid have fewer neighbors, might induce patterns in the trajectory taken by the value function. Nevertheless, closer inspection would be necessary to fully explain such behavior.

In the context of kernel-based methods, Ormoneit and Sen (2002) discuss the "biasvariance tradeoff" induced by generalization widths. Munos and Moore (2002), and Sherstov and Stone (2005), devise schemes for setting different generalization widths in different parts of the state space. Our parameterized learning problem becomes a valuable testbed to evaluate this line of work, which our results hint needs attention.

\subsection{Sequencing Sarsa and CMA-ES}

In all our experiments, we find that CMA-ES is significantly slower to learn than Sarsa. In settings with high values of $\sigma$ and low values of $\chi$, CMA-ES outperforms Sarsa mainly because Sarsa reaches a lower asymptote, and not because CMA-ES has any steeper a learning curve. In our last set of experiments, we examine whether CMA-ES can be given a boost by initializing it with a policy learned by Sarsa. We abbreviate the resulting sequencing algorithm "Seq". Since in our experiments, Sarsa and CMA-ES are constrained to share a common representation, a straightforward way to initialize CMA-ES with a policy learned using Sarsa is to set its initial weights to those learned by Sarsa. Although a raw transfer of weights is not always applicable across different representations, we conjecture that the resulting technique can still offer insights about synthesizing the merits of VF and PS methods. Guestrin et al. (2002) adopt a similar scheme in a multiagent task to transfer weights learned using LSPI to initialize a policy gradient method. In their experiments, the policy implemented is "softmax" over the learned action values; in our experiments, CMA-ES inherits a greedy policy.

In principle the method-specific parameters of Seq include all of the method-specific parameters of Sarsa $\left(\lambda, \alpha_{0}, \epsilon_{0}, \theta_{0}\right)$ and CMA-ES (\#trials, \#gens), along with the number of 
episodes at which the transfer of weights is effected from Sarsa to CMA-ES. By definition, then, Seq* would always perform at least as well as the better of Sarsa* and CMA-ES* (by running Sarsa* for 50,000 or for 0 episodes, as appropriate). Rather than search for and evaluate this best instance of Seq, we constrain Seq to (a) use Sarsa* and CMA-ES*, each optimized independently for 50,000 episodes, as its constituents; and (b) transfer weights from Sarsa* to CMA-ES* after 2,500 episodes of training. Thus, Seq essentially amounts to running CMA-ES*, but starting from a potentially useful initialization. The initial variance along each parameter to be optimized by CMA-ES* after the switch is set to the overall variance of the weights themselves.

Figure 14 compares Seq with Sarsa* and CMA-ES* under problem settings in which $\sigma$ and $\chi$ are varied. Under all settings, we find that Seq performs at least as well as CMA-ES, if not marginally better. Thorough "head-to-head" comparisons between the three methods are plotted in Fig. 15. Each plot therein compares two of the methods. For specified settings of $\sigma$ and $\chi$, the method registering higher performance is marked if the evidence is statistically significant (p-value $<0.01$ ). Figure 15(a) identifies regions of the problem space suiting Sarsa* and CMA-ES*. From Fig. 15(b), we observe that Seq marginally extends the territory

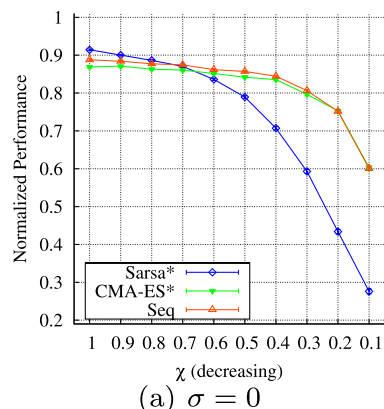

(a) $\sigma=0$

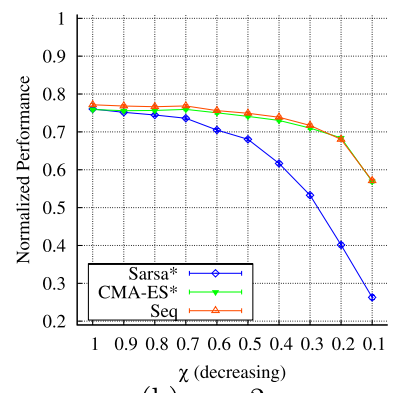

(b) $\sigma=2$

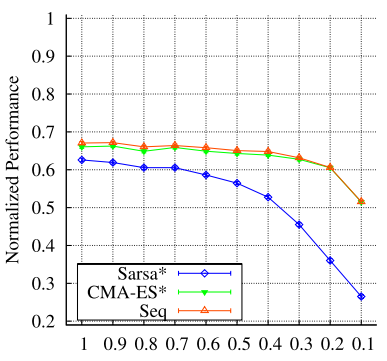

$\chi$ (decreasing)

(c) $\sigma=4$

Fig. $14[s=10, p=0.2, w=5$.] Sarsa*, CMA-ES*, and Seq compared at different settings of $\sigma$ and $\chi$. Sarsa* and CMA-ES* are optimized independently at each problem setting; Seq combines the methods thus tuned (with no further optimization), transferring weights from Sarsa to CMA-ES after 2,500 training episodes

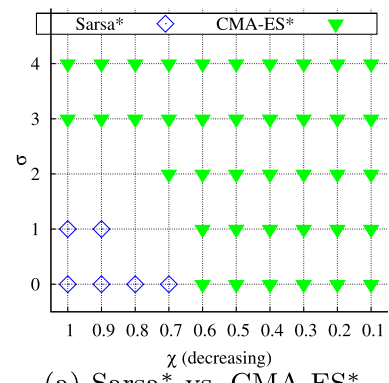

(a) Sarsa* vs. CMA-ES*

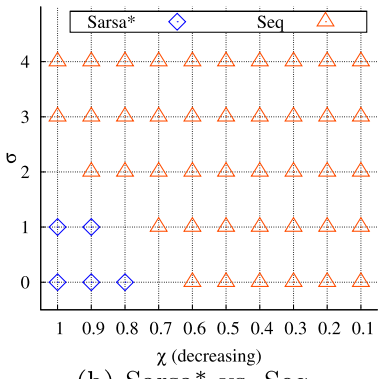

(b) Sarsa* vs. Seq

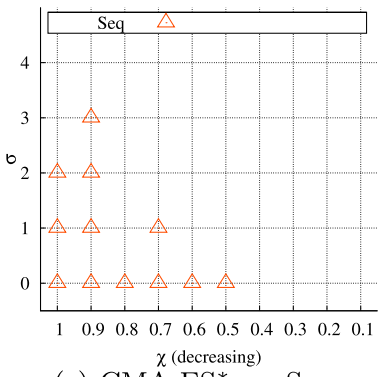

(c) CMA-ES* vs. Seq

Fig. $15[s=10, p=0.2, w=5$.] Three plots showing pairwise comparisons between Sarsa*, CMA-ES*, and Seq at different settings of $\sigma$ and $\chi$. At each reported setting, one of the methods is indicated if with statistical significance $(p<0.01)$, it achieves a higher performance than the other. At some settings, the methods cannot be thus separated; note that in plot (c), CMA-ES does not outperform Seq at any of the reported settings of $\sigma$ and $\chi$ 
claimed by CMA-ES*. Indeed Seq outperforms CMA-ES* in regions where $\sigma$ is low and $\chi$ is high, and performs at least as well in the remainder of the problem configurations (Fig. 15(c)).

As discussed in Sect. 4.2, CMA-ES requires several times the training time of Sarsa to achieve comparable performance. The experiments reported here suggest that when time is constrained, and yet CMA-ES can outshine Sarsa, CMA-ES can be further improved by seeding it with a policy learned by Sarsa. In related work (Kalyanakrishnan and Stone 2007), we demonstrate the effectiveness of Seq on Keepaway (Stone et al. 2005), a popular RL benchmarking task. In Keepaway, the function approximator used is a neural network, whose weights are initially learned using Sarsa(0), and then transferred to CEM (de Boer et al. 2005). It indeed seems very relevant to extend the entire range of experiments we have presented in this article to a complex domain such as Keepaway. The main challenge in such an exercise would be the sheer time taken for running experiments. Nevertheless, we do hope that future research will extend our current set of experiments into more complex and realistic domains.

\section{Discussion}

The extensive suite of experiments reported in Sect. 4 uncover several interesting patterns characterizing the interaction between problem parameters and method-specific parameters in the context of sequential decision making from experience. In this section, we highlight some of the main questions thereby brought to relevance.

Generalization Our results consistently indicate that generalization and function approximation significantly alter the landscape while evaluating learning algorithms, in particular those from VF. For instance, Sect. 4.2 presents conclusive evidence that ExpSarsa suffers more severely due to the bias introduced by generalization than either Sarsa or Q-learning. Section 4.6 brings into focus an irregular-yet systematic-pattern in the performance of Sarsa as the tile width $w$ is increased. Interestingly this pattern is correlated with the best eligibility trace settings. To the best of our knowledge, generalization has not been given explicit attention in the context of PS methods. Our results show that generalization can benefit PS methods, too, when the training duration is short.

As motivated in Sect. 1, generalization is necessary in nearly every practical application of RL. Thus, the importance of understanding its effects on algorithms cannot be understated. In future work, we hope to use our experimental framework to probe more deeply into this subject.

Optimistic initialization Of special significance among the ramifications of learning with generalization is its effect on the common practice of optimistic initialization. Optimistic initialization (of action values) has long been employed as a mechanism to promote exploration. In the context of finite MDPs, elegant proofs of convergence of VF methods have been derived on the basis of optimistic initial values (Even-Dar and Mansour 2001; Szita and Lốrincz 2008). Grześ and Kudenko (2009) provide experimental justification, again on finite (or suitably discretized) MDPs, for schemes that refine the basic optimistic initialization framework.

Our experiments in Sect. 4.2 convey that optimistic initialization is only effective in the fully tabular case: for $w>1$, the error introduced into TD updates by high action 
values invariably degrades the performance of VF methods. A question that arises in response is how we may initialize action values when learning with generalization. Research in this direction appears particularly relevant to algorithms that are guaranteed to reach fixed points under linear function approximation (Perkins and Precup 2003; Sutton et al. 2009; Maei et al. 2010). Which reasonable strategies for setting initial weights would profit such methods the most?

Meta-learning and algorithm portfolio design The term "meta-learning" (Vilalta and Drissi 2002) describes the enterprise of (a) characterizing the strengths and weaknesses of learning methods vis-à-vis problem characteristics, with the intent of (b) designing adaptive schemes that, given a problem, apply the method best suited for it (Brodley 1995; Pfahringer et al. 2000). Similarly, "algorithm portfolio" methods (Gomes and Selman 2001) rely on applying several candidate algorithms to a problem (either in series or in parallel) before identifying the most effective choice or combination. While existing work in these areas has largely been in the context of supervised learning and search (Leyton-Brown et al. 2003; $\mathrm{Xu}$ et al. 2008), our article is motivated by the meta-learning problem within sequential decision making.

Our experiments clearly show that there is a need for meta-learning within RL. For example, Fig. 11(c) succinctly conveys that Sarsa outperforms CMA-ES when the expressiveness of function approximation is above a certain threshold, but that the opposite is true below the threshold. Indeed our experiments unearth several other strengths and weaknesses of methods within the VF and PS classes. Additionally we provide a hybrid algorithm, Seq, as a useful step towards combining the strengths of these methods. Our results are validated on a parameterized learning problem that is specifically designed to implement a methodology for meta-learning within RL. We believe that this methodology can support the eventual development of effective algorithm portfolio designs for sequential decision making, which currently appears a rather formidable undertaking.

Automatic parameter tuning While meta-learning operates at the macro scale of choosing between methods, our experiments also underscore the gains obtained at a micro scale by tuning method-specific parameters. In this work, we have employed a search technique to optimize method-specific parameters such as learning rates and population sizes. In practice, an agent would need to automatically tune these parameters while learning. In the context of PS methods, it is worth repeating that we find existing code for CMA-ES quite adept in automatically setting and tuning several internal parameters in the algorithm. For VF methods, techniques for tuning learning rates (Sutton and Singh 1994; George and Powell 2006; Hutter and Legg 2008) and eligibility traces (Downey and Scanner 2010) have predominantly been derived and validated for the case of finite MDPs. Our parameterized learning problem serves as an excellent mechanism for prototyping adaptive schemes at more general settings involving function approximation and partial observability.

Learning and representation With the purpose of solely comparing the "learning" behavior of VF and PS methods - how they adapt a set of weights - in this study, we have forced them to share a fixed, common representation. In particular we adopt a linear function approximation scheme, whose expressiveness and generalization can be carefully controlled. Our results show that VF and PS methods dominate at different settings of these problem parameters. 
While this approach facilitates sound experimental methodology, it must be noted that in general the greatest success can be achieved by adapting the representation itself while learning (Whiteson and Stone 2006). Indeed Cobb and Bock (1994) argue that representations favoring an expert agent might be unfavorable for an agent beginning to learn. Integrating learning with adaptive representation is yet another area of future work that our parameterized learning problem enables. In pursuing such work, we would treat $\chi$ and $w$ as internal to the learning agent, rather than extraneous. The agent could potentially adapt these representational aspects by applying methods from feature selection (Kolter and $\mathrm{Ng} \mathrm{2009;}$ Petrik et al. 2010), structure learning (Degris et al. 2006; Diuk et al. 2009) and manifold learning (Mahadevan 2009).

In taking steps toward the automated application of RL methods to problems, the issues discussed above are all relevant to consider. We hope that future work will make progress along all these directions by extending the ideas presented in this article.

\section{Related work}

In this section, we discuss related work in the context of parameterized learning problems and empirical evaluations in RL.

In an early article, Cohen and Howe (1988) consider the strong coupling that exists in many disciplines of AI between problem types and method instances. While formulating guidelines for the evaluation of methodological contributions to the field, they argue the need to precisely characterize the set of problems on which a method is expected to be successful, and symmetrically, the approaches that are likely to succeed on a given class of problems. As mentioned in Sect. 1, Langley (1988) makes a similar observation in the specific context of machine learning.

Parameterized learning problems have been used in the literature to study the effects of factors such as dimensionality and noise. For example, Spall (2003) extensively uses the "Rosenbrock" function while comparing the performance of optimization algorithms in his textbook. The "Sphere" function discussed by Beyer (2000) has served as a standard benchmark for evolutionary algorithms.

The work within the RL literature that is philosophically closest to the contribution of this article is the notion of "generalized environments" proposed by (Whiteson et al. 2011). Here, too, the authors argue against "environment overfitting", whereby methods tend to get evaluated on problems that favor them, but the broader scope of their applicability, especially their weaknesses, are not easy to gauge. A generalized environment represents a formally defined distribution of environments: the objective is to develop methods that perform well over the entire distribution. Whereas the motivation for generalized environments comes from realistic tasks such as helicopter control and Tetris, the apparatus developed in this article examines the performance of learning methods in a carefully-designed, controllable, abstract setting. Our results underscore that qualitatively different learning methods excel in different regions of the problem space, while teasing apart effects that method-specific parameters introduce.

The empirical approach we adopt in this article to characterize the interactions between learning problems and methods is complemented by theoretical formulations designed with a similar objective. Littman (1993) characterizes agents and environments based on the amount of memory they can use and the length of the horizon for which they seek to optimize rewards. He then considers several interesting classes of problems-for example, those with 
nonstationary environments - that fit within this formalization. Ratitch and Precup (2003) define environmental properties such as state transition entropy and forward controllability, and investigate how these properties bear on the exploration strategy of a learning agent. To the best of our knowledge, generalization and function approximation have not been addressed through similar theoretical formulations.

The main difference between our comparative study and others in the RL literature is that our parameterized learning problem enables us to evaluate the effects of individual parameters while keeping others fixed. For example, in most related studies, methods typically use different function approximation schemes, thereby introducing an additional qualification for comparing them. Also, our formulation allows us to control problem parameters continuously along a scale from "high" to "low"; in the studies we shortly list, comparisons are typically between two or three distinct task settings. In this sense, our article answers the call put forth by Togelius et al. (2009) for parameterizable benchmarks, and affirms their basic conjectures on the strengths and weaknesses of "ontogenetic" (similar to VF) and "phylogenetic" (similar to PS) methods. In addition our results shed light on hitherto unexplored questions such as the effects of optimistic initialization when used in conjunction with generalization. We proceed to briefly list a number of studies comparing RL algorithms.

Moriarty et al. (1999) apply a suite of "Evolutionary Algorithms for Reinforcement Learning" (EARL) to a simple grid-world MDP, and compare results with Q-learning. Policies are represented using lists of rules or neural networks, which are evolved using standard genetic operators. The main conclusion of their study is that EARL is more suited to tasks with large state spaces (but represented compactly), tasks with incomplete state information, and tasks with nonstationary returns. Whiteson et al. (2010) undertake a comparative study between Sarsa(0) and NEAT (Stanley 2004), a policy search method. These methods are compared on the benchmark tasks of Keepaway soccer (Stone et al. 2005) and Mountain Car (Sutton and Barto 1998). Their findings are that sensor noise affects the final performance of Sarsa( 0 ) more than NEAT, and indeed that stochasticity has the opposite effect, as policy evaluations under NEAT become more noisy.

Heidrich-Meisner and Igel (2008a) compare the natural actor-critic method with CMAES on a pole-balancing task. Both methods are "variable metric"; i.e., they are insensitive to linear transformations of the parameter space. The methods achieve comparable results, but CMA-ES is found to be less sensitive to initial values for the policy, which has a small number of parameters. Similar results are registered in the noisy Mountain Car task (HeidrichMeisner and Igel 2008b). A more extensive suite of comparisons on single and double polebalancing tasks (with hidden state) is carried out by Gomez et al. (2008). The methods compared are evolutionary algorithms such as CoSyNE, NEAT, ESP, and SANE, along with Q-learning, Sarsa, recurrent policy gradient, random weight guessing, and "Value and Policy Search" (VAPS) (Baird and Moore 1999). The findings reinforce the expectation that under partial observability, evolutionary algorithms dominate model-free value functionbased methods such as Q-learning and Sarsa. As with our Seq algorithm, a number of approaches, both empirical and theoretical, have been proposed to combine the strengths of qualitatively different learning approaches (Guestrin et al. 2002; Konda and Tsitsiklis 2003; Whiteson and Stone 2006).

\section{Summary}

A large number of reinforcement learning (RL) tasks we face in the real world cannot be modeled and solved exactly as finite MDPs, which support theoretical guarantees such as 
convergence and optimality. The objective of learning in these tasks has to be rescaled to realizing policies with "high" expected long-term reward in a "sample efficient" manner, as determined empirically. Consequently it becomes necessary to characterize the performance of different learning methods on different problems.

As a framework to conduct empirical studies in RL, we introduce parameterized learning problems, in which the factors influencing the performance of a learning algorithm can be controlled systematically through targeted studies. The main merits of our experimental methodology are that (a) the designed task and learning framework are easy to understand; (b) we may examine the effect of subsets of problem parameters while keeping others fixed; (c) we can benchmark learned policies against optimal behavior; and (d) the learning process can be executed in a relatively short duration of time, thereby facilitating extensive experimentation.

In particular we design a parameterized learning problem to evaluate the effects of partial observability and function approximation, which characterize a sizeable fraction of realistic RL tasks. On this problem, we evaluate various methods from the classes of on-line value function-based (VF) methods and policy search (PS) methods. Through a series of carefully-designed experiments, we obtain clear patterns separating the learning methods considered. A novel aspect of our study is a search procedure that enables us to find the best method-specific parameters (such as learning rates and population sizes) for a given problem instance. Largely made possible by the relative simplicity of our simulation, the search procedure uncovers interesting patterns relating problem instances and method-specific parameters.

Within the VF class, we find that Sarsa and Q-learning perform better than Expected Sarsa (ExpSarsa) when learning with generalization and function approximation. Within the PS class, we find that CMA-ES and the cross-entropy method (CEM) achieve significantly better performance than a genetic algorithm (GA); CMA-ES is more robust to its parameter settings than CEM. Comparing Sarsa (from VF) and CMA-ES (from PS), we find that the former enjoys a higher speed of learning, and also better asymptotic performance, when the learner is provided an expressive representation. On the other hand, CMA-ES is significantly more robust to severely deficient representations. Both methods suffer noticeably when state noise is added; their relative performance is additionally determined by the width of generalization in the representation.

Our experiments highlight several promising lines of inquiry involving generalization, representation, meta-learning, initial weight settings, and parameter tuning. By contributing a novel evaluation methodology and a preliminary set of results obtained using it, our article is oriented towards ultimately constructing a field guide for the application of RL methods in practice. The experiments we have reported in this article are part of an ongoing study, which we plan to extend to other classes of methods, including model-based and batch methods, actor-critic architectures, and policy gradient techniques.

Acknowledgements We are very grateful to the anonymous reviewers of this article, to the special issue editors, and especially to Michael L. Littman, for raising several important questions concerning our experiments and claims. The article has benefited significantly from their advice. We thank Harm van Seijen for sharing several useful insights about the methods compared in Sect. 4.2.1, and Juhyun Lee for proofreading the article. This work has taken place in the Learning Agents Research Group (LARG) at the Artificial Intelligence Laboratory, The University of Texas at Austin. LARG research is supported in part by grants from the National Science Foundation (CNS-0615104 and IIS-0917122), the Office of Naval Research (N0001409-1-0658), and the Federal Highway Administration (DTFH61-07-H-00030). 


\section{Appendix A: Effect of $\alpha_{0}$ and $\epsilon_{0}$ on methods in VF}

The plots below show the effect of the initial learning rate $\alpha_{0}$ and exploration rate $\epsilon_{0}$ on the learned performance of different methods in VF. Intensity ranges are indicated to the right of each plot. Under $I_{1}$, all the methods use $\theta_{0}=10$; under $I_{2}$, they use $\theta_{0}=0.5$; under $I_{3}$, they use $\theta_{0}=5$. We observe that under instance $I_{1}$, Sarsa(0), Q-learning(0), and $\operatorname{ExpSarsa(0)}$ all achieve normalized performance values close to 1 . Under $I_{2}$, ExpSarsa(0) and $\operatorname{ExpSarsa(1)}$ perform best at low values of $\alpha_{0}$ and $\epsilon_{0}$.

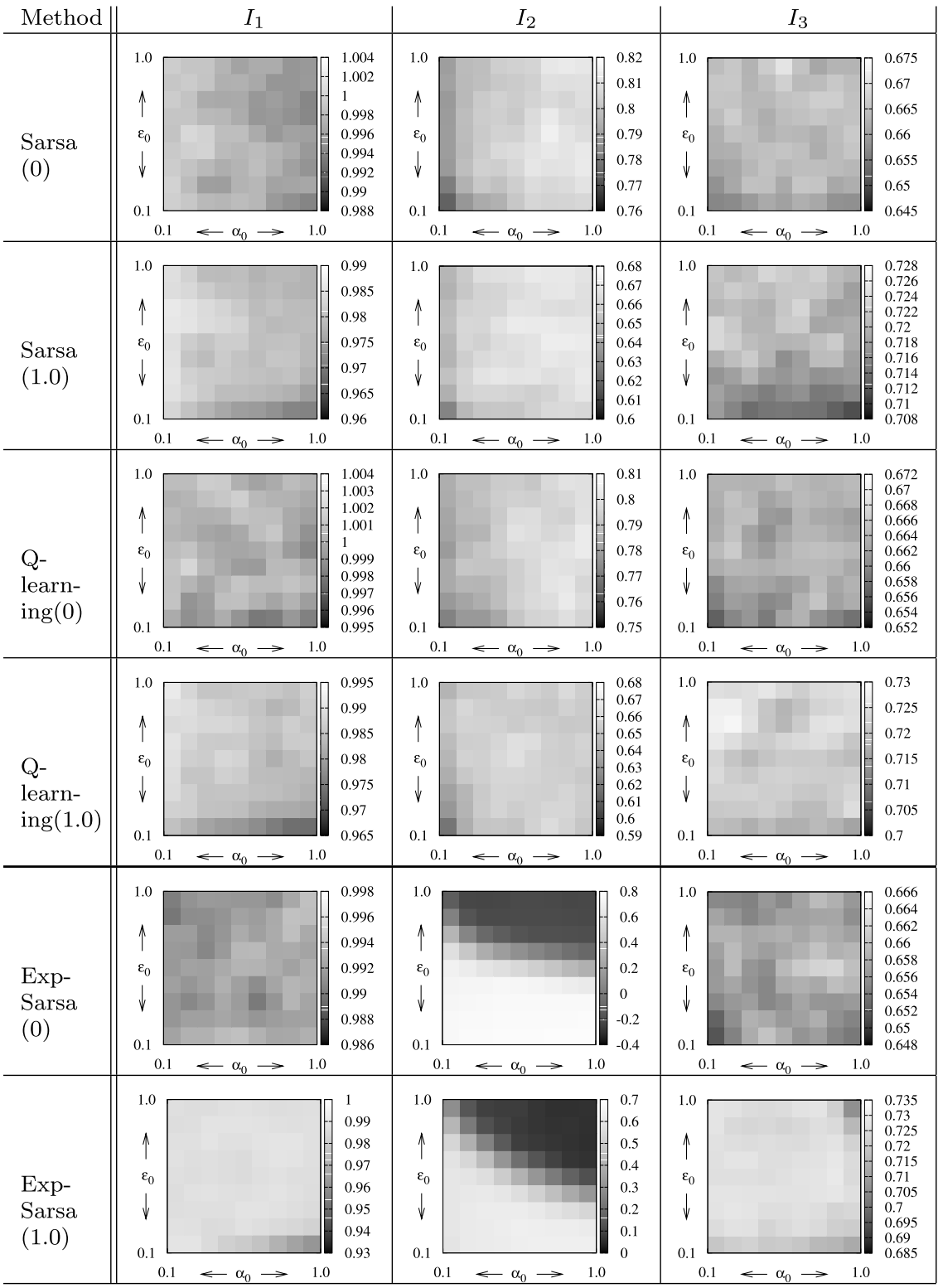




\section{Appendix B: Effect of \#trials and \#gens on Methods in PS}

The plots below show the effect of \#trials and \#gens on the performance of different policy search methods. Intensity ranges are indicated to the right of each plot. Under RWG, only \#trials is varied (\#gens $=\frac{50000}{\# \text { ttrials }}$ ); the mean performance is plotted with one standard error. The methods all show noticeable variance in performance over the ranges plotted, underscoring the need for careful tuning. For all methods, the highest performance is under problem instance $I_{2}$. We see that CMA-ES performs better on average, over the parameter ranges plotted, than both CEM and GA.

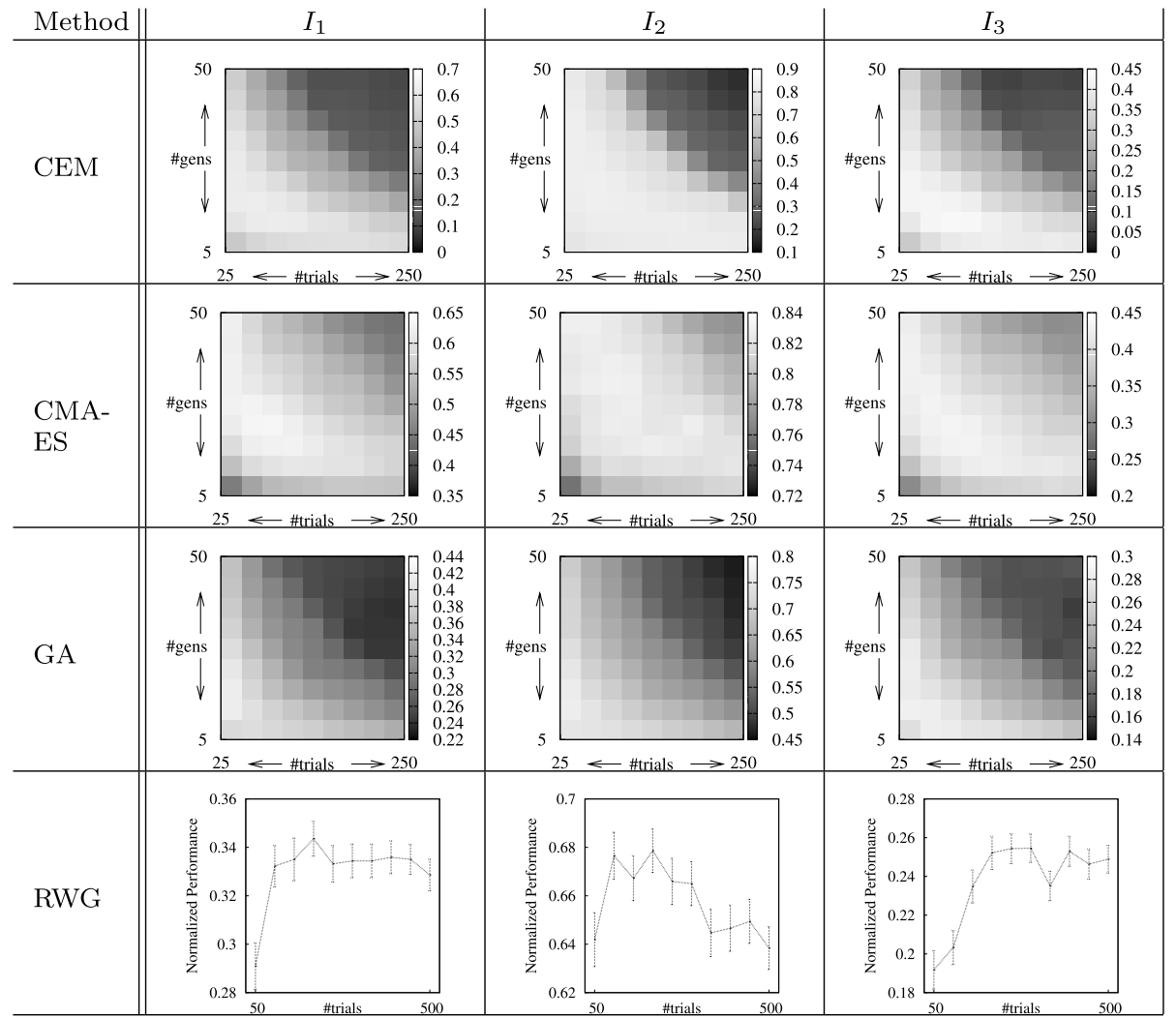

\section{References}

Albus, J. S. (1981). Brains, behavior and robotics. New York: McGraw-Hill.

Åström, K. J. (1965). Optimal control of Markov processes with incomplete state information. Journal of Mathematical Analysis and Applications, 10, 174-205.

Baird, L., \& Moore, A. (1999). Gradient descent for general reinforcement learning. In M. J. Kearns, S. A. Solla, \& D. A. Cohn (Eds.), Advances in neural information processing systems 11 (NIPS 1998) (pp. 968-974). Cambridge: MIT Press.

Bakker, B., Zhumatiy, V., Gruener, G., \& Schmidhuber, J. (2003). A robot that reinforcement-learns to identify and memorize important previous observations. In Proceedings of the 2003 IEEE/RSJ international conference on intelligent robots and systems (IROS 2003) (pp. 430-435). New York: IEEE Press. 
Banko, M., \& Brill, E. (2001). Scaling to very very large corpora for natural language disambiguation. In Proceedings of 39th annual meeting of the association for computational linguistics (ACL 2001) (pp. 26-33). Association for Computational Linguistics.

Bauer, E., \& Kohavi, R. (1999). An empirical comparison of voting classification algorithms: bagging, boosting, and variants. Machine Learning, 36(1-2), 105-139.

Baxter, J., \& Bartlett, P. L. (2001). Infinite-horizon policy-gradient estimation. The Journal of Artificial Intelligence Research, 15, 319-350.

Bellman, R. (1957). Dynamic programming, 1st ed. (p. 1). Princeton: Princeton University Press.

Bertsekas, D. P., \& Tsitsiklis, J. N. (1996). Neuro-dynamic programming. Nashua: Athena Scientific.

Beyer, H.-G. (2000). Evolutionary algorithms in noisy environments: theoretical issues and guidelines for practice. Computer Methods in Applied Mechanics and Engineering, 186(2-4), 239-267.

Brafman, R. I., \& Tennenholtz, M. (2003). R-MAX — a general polynomial time algorithm for near-optimal reinforcement learning. Journal of Machine Learning Research, 3, 213-231.

Breiman, L. (2001). Random forests. Machine Learning, 45(1), 5-32.

Brodley, C. E. (1995). Recursive automatic bias selection for classifier construction. Machine Learning, 20(12), 63-94.

Caruana, R., \& Niculescu-Mizil, A. (2006). An empirical comparison of supervised learning algorithms. In W. W. Cohen \& A. Moore (Eds.), Proceedings of the twenty-third international conference on machine learning (ICML 2006) (pp. 161-168). New York: ACM.

Caruana, R., Karampatziakis, N., \& Yessenalina, A. (2008). An empirical evaluation of supervised learning in high dimensions. In W. W. Cohen, A. McCallum, \& S. T. Roweis (Eds.), Proceedings of the twenty-fifth international conference on machine learning (ICML 2008) (pp. 96-103). New York: ACM.

Cassandra, A. R., Kaelbling, L. P., \& Littman, M. L. (1994). Acting optimally in partially observable stochastic domains. In Proceedings of the twelfth national conference on artificial intelligence (AAAI 1994) (pp. 1023-1028). Menlo Park: AAAI Press.

Chrisman, L. (1992). Reinforcement learning with perceptual aliasing: The perceptual distinctions approach. In W. R. Swartout (Ed.), Proceedings of the tenth national conference on artificial intelligence (AAAI 1992) (pp. 183-188). Menlo Park: AAAI Press.

Cobb, H. G. (1992). Inductive biases in a reinforcement learner. Technical report AIC-92-013, Navy Center for Applied Research in Artificial Intelligence, Washington DC, USA.

Cobb, H. G., \& Bock, P. (1994). Using a genetic algorithm to search for the representational bias of a collective reinforcement learner. In Y. Davidor, H.-P. Schwefel, \& R. Männer (Eds.), Proceedings of the third conference on parallel problem solving from nature (PPSN III) (pp. 576-587). Berlin: Springer.

Cohen, P. R., \& Howe, A. E. (1988). How evaluation guides AI research: the message still counts more than the medium. The AI Magazine, 9(4), 35-43.

Crites, R. H., \& Barto, A. G. (1996). Improving elevator performance using reinforcement learning. In D. S. Touretzky, M. Mozer, \& M. E. Hasselmo (Eds.), Advances in neural information processing systems 8 (NIPS 1995) (pp. 1017-1023). Cambridge: MIT Press.

Dayan, P., \& Sejnowski, T. J. (1994). TD $(\lambda)$ converges with probability 1. Machine Learning, 14, $295-301$.

de Boer, P.-T., Kroese, D. P., Mannor, S., \& Rubinstein, R. Y. (2005). A tutorial on the cross-entropy method. Annals of Operation Research, 134(1), 19-67.

Degris, T., Sigaud, O., \& Wuillemin, P.-H. (2006). Learning the structure of factored Markov Decision Processes in reinforcement learning problems. In W. W. Cohen \& A. Moore (Eds.), Proceedings of the twenty-third international conference on machine learning (ICML 2006) (pp. 257-264). New York: ACM.

Diuk, C., Li, L., \& Leffler, B. R. (2009). The Adaptive $k$-Meteorologists problem and its application to structure learning and feature selection in reinforcement learning. In A. P. Danyluk, L. Bottou, \& M. L. Littman (Eds.), Proceedings of the twenty-sixth annual international conference on machine learning (ICML 2009) (pp. 249-256). New York: ACM.

Downey, C., \& Scanner, S. (2010). Temporal difference Bayesian model averaging: A Bayesian perspective on adapting lambda. In J. Fürnkranz, \& T. Cohn (Joachims) (Eds.), Proceedings of the twenty-seventh international conference on machine learning (pp. 311-318). Madison: Omnipress.

Even-Dar, E., \& Mansour, Y. (2001). Convergence of optimistic and incremental Q-Learning. In T. G. Dietterich, S. Becker, \& Z. Ghahramani (Eds.), Advances in neural information processing systems 14 (NIPS 2001) (pp. 1499-1506). Cambridge: MIT Press.

Finnsson, H., \& Björnsson, Y. (2008). Simulation-based approach to General Game Playing. In D. Fox \& C. P. Gomes (Eds.), Proceedings of the twenty-third AAAI conference on artificial intelligence (AAAI 2008) (pp. 259-264). Menlo Park: AAAI Press.

Freund, Y., \& Schapire, R. E. (1996). Experiments with a new boosting algorithm. In L. Saitta (Ed.), Proceedings of the thirteenth international conference on machine learning (ICML 1996) (pp. 148-156). Los Altos: Kaufmann. 
Gabel, T., Riedmiller, M., \& Trost, F. (2009). A case study on improving defense behavior in soccer simulation 2D: The NeuroHassle approach. In L. Iocchi, H. Matsubara, A. Weitzenfeld, \& Z. Changjiu (Eds.), RoboCup 2008: robot soccer world cup XII (pp. 61-72). Berlin: Springer.

George, A. P., \& Powell, W. B. (2006). Adaptive stepsizes for recursive estimation with applications in approximate dynamic programming. Machine Learning, 65(1), 167-198.

Gomes, C. P., \& Selman, B. (2001). Algorithm portfolios. Artificial Intelligence, 126(1-2), 43-62.

Gomez, F., Schmidhuber, J., \& Miikkulainen, R. (2008). Accelerated neural evolution through cooperatively coevolved synapses. Journal of Machine Learning Research, 9, 937-965.

Gomez, F. J., \& Miikkulainen, R. (1999). Solving non-Markovian control tasks with neuro-evolution. In T. Dean (Ed.), Proceedings of the sixteenth international joint conference on artificial intelligence (IJCAI 1999) (pp. 1356-1362). Los Altos: Kaufmann.

Gomez, F. J., \& Miikkulainen, R. (2003). Active guidance for a finless rocket using neuroevolution. In E. Cantú-Paz, J. A. Foster, K. Deb, L. Davis, R. Roy, U.-M. O’Reilly, H.-G. Beyer, R. K. Standish, G. Kendall, S. W. Wilson, M. Harman, J. Wegener, D. Dasgupta, M. A. Potter, A. C. Schultz, K. A. Dowsland, N. Jonoska, \& J. F. Miller (Eds.), Proceedings of the genetic and evolutionary computation conference (GECCO 2003) (pp. 2084-2095). Berlin: Springer.

Grześ, M., \& Kudenko, D. (2009). Improving optimistic exploration in model-free reinforcement learning. In M. Kolehmainen, P. J. Toivanen, \& B. Beliczynski (Eds.), Proceedings of the ninth international conference on adaptive and natural computing algorithms (ICANNGA 2009) (pp. 360-369). Berlin: Springer.

Guestrin, C., Lagoudakis, M. G., \& Parr, R. (2002). Coordinated reinforcement learning. In C. Sammut \& A. G. Hoffman (Eds.), Proceedings of the nineteenth international conference on machine learning (ICML 2002) (pp. 227-234). Los Altos: Kaufmann.

Guez, A., Vincent, R. D., Avoli, M., \& Pineau, J. (2008). Adaptive treatment of epilepsy via batch-mode reinforcement learning. In D. Fox \& C. P. Gomes (Eds.), Proceedings of the twenty-third AAAI conference on artificial intelligence (AAAI 2008) (pp. 1671-1678). Menlo Park: AAAI Press.

Hansen, N. (2009). The CMA evolution strategy: a tutorial. http://www.lri.fr/ hansen/cmatutorial.pdf.

Hansen, N., Niederberger, A. S., Guzzella, L., \& Koumoutsakos, P. (2009). A method for handling uncertainty in evolutionary optimization with an application to feedback control of combustion. IEEE Transactions on Evolutionary Computation, 13(1), 180-197.

Heidrich-Meisner, V., \& Igel, C. (2008a). Similarities and differences between policy gradient methods and evolution strategies. In M. Verleysen (Ed.), Proceedings of the sixteenth European symposium on artificial neural networks (ESANN 2008) (pp. 149-154). Evere: D-side Publication.

Heidrich-Meisner, V., \& Igel, C. (2008b). Variable metric reinforcement learning methods applied to the noisy mountain car problem. In S. Girgin, M. Loth, R. Munos, P. Preux, \& D. Ryabko (Eds.), Recent advances in reinforcement learning: eighth European workshop (EWRL 2008) (pp. 136-150). Berlin: Springer.

Heidrich-Meisner, V., \& Igel, C. (2009). Hoeffding and Bernstein races for selecting policies in evolutionary direct policy search. In A. P. Danyluk, L. Bottou, \& M. L. Littman (Eds.), Proceedings of the twentysixth international conference on machine learning (ICML 2009) (pp. 401-408). New York: ACM.

Hutter, M., \& Legg, S. (2008). Temporal difference updating without a learning rate. In J. C. Platt, D. Koller, Y. Singer, \& S. T. Roweis (Eds.), Advances in neural information processing systems 20 (NIPS 2007) (pp. 705-712). Cambridge: MIT Press.

İpek, E., Mutlu, O., Martínez, J., \& Caruana, R. (2008). Self-optimizing memory controllers: a reinforcement learning approach. In Proceedings of the thirty-fifth international symposium on computer architecture (ISCA 2008) (pp. 39-50). New York: IEEE Press.

James, M. R., \& Singh, S. (2009). SarsaLandmark: an algorithm for learning in POMDPs with landmarks. In C. Sierra, C. Castelfranchi, K. S. Decker, \& J. S. Sichman (Eds.), Proceedings of the eighth international conference on autonomous agents and multiagent systems (AAMAS 2009) (pp. 585-591). IFAAMAS.

Kakade, S. (2001). A natural policy gradient. In T. G. Dietterich, S. Becker, \& Z. Ghahramani (Eds.), Advances in neural information processing systems 14 (NIPS 2001) (pp. 1531-1538). Cambridge: MIT Press.

Kalyanakrishnan, S., \& Stone, P. (2007). Batch reinforcement learning in a complex domain. In E. H. Durfee, M. Yokoo, M. N. Huhns, \& O. Shehory (Eds.), Proceedings of the sixth international joint conference on autonomous agents and multiagent systems (AAMAS 2007) (pp. 650-657). IFAAMAS.

Kearns, M., \& Singh, S. (2002). Near-optimal reinforcement learning in polynomial time. Machine Learning, 49(2-3), 209-232.

Kohl, N., \& Stone, P. (2004). Machine learning for fast quadrupedal locomotion. In D. L. McGuinness \& G. Ferguson (Eds.), Proceedings of the nineteenth national conference on artificial intelligence (AAAI 2004) (pp. 611-616). Menlo Park: AAAI Press. 
Kolter, J. Z., \& Ng, A. Y. (2009). Regularization and feature selection in least-squares temporal difference learning. In A. P. Danyluk, L. Bottou, \& M. L. Littman (Eds.), Proceedings of the twenty-sixth annual international conference on machine learning (ICML 2009) (pp. 521-528). New York: ACM.

Konda, V. R., \& Tsitsiklis, J. N. (2003). On actor-critic algorithms. SIAM Journal on Control and Optimization, 42(4), 1143-1166.

Kwok, C., \& Fox, D. (2004). Reinforcement learning for sensing strategies. In Proceedings of the 2004 IEEE/RSJ international conference on intelligent robots and systems (ICRA 2004) (pp. 3158-3163). New York: IEEE Press.

Lagoudakis, M. G., \& Parr, R. (2003). Least-squares policy iteration. Journal of Machine Learning Research, 4, 1107-1149.

Langley, P. (1988). Machine learning as an experimental science. Machine Learning, 3(1), 5-8.

Langley, P., \& Pendrith, M. (1998). Symposium on applications of reinforcement learning: final report for NSF Grant IIS-9810208. Technical report. Institute for the Study of Learning and Expertise.

Lee, H., Shen, Y., Yu, C.-H., Singh, G., \& Ng, A. Y. (2006). Quadruped robot obstacle negotiation via reinforcement learning. In Proceedings of the 2006 IEEE international conference on robotics and automation (ICRA 2006) (pp. 3003-3010). New York: IEEE Press.

Leyton-Brown, K., Nudelman, E., Andrew, G., McFadden, J., \& Shoham, Y. (2003). Boosting as a metaphor for algorithm design. In F. Rossi (Ed.), Proceedings of the ninth international conference on principles and practice of constraint programming (CP 2003) (pp. 899-903). Berlin: Springer.

Lin, L.-J. (1992). Self-improving reactive agents based on reinforcement learning, planning and teaching. Machine Learning, 8(3-4), 293-321.

Lin, L.-J., \& Mitchell, T. M. (1993). Reinforcement learning with hidden states. In J.-A. Meyer, H. L. Roitblat, \& S. W. Wilson (Eds.), From animals to animats 2 (pp. 271-280). Cambridge: MIT Press.

Littlestone, N. (1987). Learning quickly when irrelevant attributes abound: a new linear-threshold algorithm. Machine Learning, 2(4), 285-318.

Littman, M. L. (1993). An optimization-based categorization of reinforcement learning environments. In J.A. Meyer, H. L. Roitblat, \& S. W. Wilson (Eds.), From animals to animats 2 (pp. 262-270). Cambridge: MIT Press.

Loch, J., \& Singh, S. (1998). Using eligibility traces to find the best memoryless policy in partially observable Markov decision processes. In J. W. Shavlik (Ed.), Proceedings of the fifteenth international conference on machine learning (ICML 1998) (pp. 323-331). Los Altos: Kaufmann.

Maei, H. R., Szepesvári, C., Bhatnagar, S., \& Sutton, R. S. (2010). Toward off-policy learning control with function approximation. In J. Fürnkranz \& T. Joachims (Eds.), Proceedings of the twenty-seventh international conference on machine learning (ICML 2010) (pp. 719-726). Madison: Omnipress.

Mahadevan, S. (2009). Learning representation and control in Markov decision processes: new frontiers. Foundations and Trends in Machine Learning, 1(4), 403-565.

McCallum, A. K. (1996). Reinforcement learning with selective perception and hidden state. Ph.D. thesis, Computer Science Department, University of Rochester.

McCallum, R. A. (1995). Instance-based utile distinctions for reinforcement learning with hidden state. In A. Prieditis \& S. J. Russell (Eds.), Proceedings of the twelfth international conference on machine learning (ICML 1995) (pp. 387-395). Los Altos: Kaufmann.

Melo, F. S., Meyn, S. P., \& Ribeiro, M. I. (2008). An analysis of reinforcement learning with function approximation. In Proceedings of the twenty-fifth international conference on machine learning (ICML 2008) (pp. 664-671). New York: ACM.

Metzen, J. H., Edgington, M., Kassahun, Y., \& Kirchner, F. (2008). Analysis of an evolutionary reinforcement learning method in a multiagent domain. In L. Padgham, D. C. Parkes, J. Müller, \& S. Parsons (Eds.), Proceedings of the seventh international joint conference on autonomous agents and multiagent systems (AAMAS 2008) (pp. 291-298). IFAAMAS.

Moriarty, D. E., Schultz, A. C., \& Grefenstette, J. J. (1999). Evolutionary algorithms for reinforcement learning. The Journal of Artificial Intelligence Research, 11, 241-276.

Munos, R., \& Moore, A. W. (2002). Variable resolution discretization in optimal control. Machine Learning, 49(2-3), 291-323.

Nevmyvaka, Y., Feng, Y., \& Kearns, M. (2006). Reinforcement learning for optimized trade execution. In W. W. Cohen \& A. Moore (Eds.), Proceedings of the twenty-third international conference on machine learning (ICML 2006) (pp. 673-680). New York: ACM.

Ng, A. Y., Kim, H. J., Jordan, M. I., \& Sastry, S. (2004). Autonomous helicopter flight via reinforcement learning. In S. Thrun, L. K. Saul, \& B. Schölkopf (Eds.), Advances in neural information processing systems 16 (NIPS 2003) Cambridge: MIT Press.

Ormoneit, D., \& Sen, S. (2002). Kernel-based reinforcement learning. Machine Learning, 49(2-3), 161-178.

Peng, J., \& Williams, R. J. (1996). Incremental multi-step Q-learning. Machine Learning, 22(1-3), $283-290$. 
Perkins, T. J., \& Pendrith, M. D. (2002). On the existence of fixed points for Q-Learning and Sarsa in partially observable domains. In C. Sammut \& A. Hoffman (Eds.), Proceedings of the nineteenth international conference on machine learning (ICML 2002) (pp. 490-497). Los Altos: Kaufmann.

Perkins, T. J., \& Precup, D. (2003). A convergent form of approximate policy iteration. In S. Becker, S. Thrun, \& K. Obermayer (Eds.), Advances in neural information processing systems 15 (NIPS 2002) (pp. 1595-1602). Cambridge: MIT Press.

Peters, J., \& Schaal, S. (2008). Reinforcement learning of motor skills with policy gradients. Neural Networks, 21(4), 682-697.

Petrik, M., Taylor, G., Parr, R., \& Zilberstein, S. (2010). Feature selection using regularization in approximate linear programs for Markov Decision Processes. In J. Fürnkranz \& T. Joachims (Eds.), Proceedings of the twenty-seventh international conference on machine learning (ICML 2010) (pp. 871-878). Madison: Omnipress.

Pfahringer, B., Bensusan, H., \& Giraud-Carrier, C. (2000). Meta-learning by landmarking various learning algorithms. In P. Langley (Ed.), Proceedings of the seventeenth international conference on machine learning (ICML 2000) (pp. 743-750). Los Altos: Kaufmann.

Pineau, J., Gordon, G. J., \& Thrun, S. (2006). Anytime point-based approximations for large POMDPs. The Journal of Artificial Intelligence Research, 27, 335-380.

Precup, D., Sutton, R. S., \& Dasgupta, S. (2001). Off-policy temporal difference learning with function approximation. In C. E. Brodley \& A. P. Danyluk (Eds.), Proceedings of the eighteenth international conference on machine learning (ICML 2001) (pp. 417-424). Los Altos: Kaufmann.

Quinlan, J. R. (1996). Bagging, boosting, and C4.5. In Proceedings of the thirteenth national conference on artificial intelligence (AAAI 1996) (pp. 725-730). Berlin: Springer.

Ratitch, B., \& Precup, D. (2003). Using MDP characteristics to guide exploration in reinforcement learning. In N. Lavrac, D. Gamberger, L. Todorovski, \& H. Blockeel (Eds.), Proceedings of the fourteenth European conference on machine learning (ECML 2003) (pp. 313-324). Berlin: Springer.

Rottmann, A., Plagemann, C., Hilgers, P., \& Burgard, W. (2007). Autonomous blimp control using modelfree reinforcement learning in a continuous state and action space. In Proceedings of the 2007 IEEE/RSJ international conference on intelligent robots and systems (IROS 2007), pages (pp. 1895-1900). New York: IEEE Press.

Rummery, G. A. (1995). Problem solving with reinforcement learning. Ph.D. thesis, Cambridge University Engineering Department, Cambridge, UK.

Rummery, G. A., \& Niranjan, M. (1994). On-line Q-learning using connectionist systems. CUED/FINFENG/TR 166, Cambridge University Engineering Department.

Sabes, P. (1993). Approximating Q-values with basis function representations. In M. Mozer, P. Smolensky, D. Touretzky, J. Elman, \& A. Weigend (Eds.), Proceedings of the 1993 connectionist models summer school (pp. 264-271). Hillsdale: Erlbaum.

Sherstov, A. A., \& Stone, P. (2005). Function approximation via tile coding: Automating parameter choice. In J.-D. Zucker \& L. Saitta (Eds.), Proceedings of the sixth international symposium on abstraction, reformulation and approximation (SARA 2005) (pp. 194-205). Berlin: Springer.

Silver, D., Sutton, R. S., \& Müller, M. (2007). Reinforcement learning of local shape in the game of Go. In M. M. Veloso (Ed.), Proceedings of the twentieth international joint conference on artificial intelligence (IJCAI 2007) (pp. 1053-1058). IJCAI.

Singh, S., \& Bertsekas, D. (1997). Reinforcement learning for dynamic channel allocation in cellular telephone systems. In M. Mozer, M. I. Jordan, \& T. Petsche (Eds.), Advances in neural information processing systems 9 (NIPS 1996) (pp. 974-980). Cambridge: MIT Press.

Singh, S., Jaakkola, T., Littman, M. L., \& Szepesvári, C. (2000). Convergence results for single-step on-policy reinforcement-learning algorithms. Machine Learning, 38(3), 287-308.

Singh, S. P., \& Sutton, R. S. (1996). Reinforcement learning with replacing eligibility traces. Machine Learning, 22(1-3), 123-158.

Singh, S. P., Jaakkola, T., \& Jordan, M. I. (1994). Learning without state-estimation in partially observable Markovian decision processes. In W. W. Cohen \& H. Hirsch (Eds.), Proceedings of the eleventh international conference on machine learning (ICML 1994) (pp. 284-292). Los Altos: Kaufmann.

Spall, J. C. (2003). Introduction to stochastic search and optimization. Hoboken: Wiley.

Stanley, K. O. (2004). Efficient evolution of neural networks through complexification. Ph.D. thesis, Department of Computer Sciences, University of Texas at Austin. Published as technical report AI-TR-04-314.

Stone, P., Sutton, R. S., \& Kuhlmann, G. (2005). Reinforcement learning for RoboCup-soccer keepaway. Adaptive Behavior, 13(3), 165-188.

Strehl, A. L., \& Littman, M. L. (2005). A theoretical analysis of model-based interval estimation. In L. De Raedt \& S. Wrobel (Eds.), Proceedings of the twenty-second international conference on machine learning (ICML 2005) (pp. 856-863). New York: ACM. 
Strehl, A. L., Li, L., Wiewiora, E., Langford, J., \& Littman, M. L. (2006). PAC model-free reinforcement learning. In W. W. Cohen \& A. Moore (Eds.), Proceedings of the twenty-third international conference on machine learning (ICML 2006) (pp. 881-888). New York: ACM.

Sutton, R. S. (1988). Learning to predict by the methods of temporal differences. Machine Learning, 3(1), 9-44.

Sutton, R. S. (1990). Integrated architectures for learning, planning, and reacting based on Approximating Dynamic Programming. In B. W. Porter \& R. J. Mooney (Eds.), Proceedings of the seventh international conference on machine learning (ICML 1990) (pp. 216-224). Los Altos: Kaufmann.

Sutton, R. S. (1996). Generalization in reinforcement learning: Successful examples using sparse coarse coding. In D. S. Touretzky, M. Mozer, \& M. E. Hasselmo (Eds.), Advances in neural information processing systems 8 (NIPS 1995) (pp. 1038-1044). Cambridge: MIT Press.

Sutton, R. S., \& Barto, A. G. (1998). Reinforcement learning: an introduction. Cambridge: MIT Press.

Sutton, R. S., \& Singh, S. P. (1994). On bias and step size in temporal-difference learning. In Proceedings of the eighth Yale workshop on adaptive and learning systems (pp. 91-96). New Haven, CT, USA. Center for Systems Science, Yale University.

Sutton, R. S., McAllester, D. A., Singh, S. P., \& Mansour, Y. (2000). Policy gradient methods for reinforcement learning with function approximation. In S. A. Solla, T. K. Leen, \& K.-R. Müller (Eds.), Advances in neural information processing systems 12 (NIPS 1999) (pp. 1057-1063). Cambridge: MIT Press.

Sutton, R. S., Maei, H. R., Precup, D., Bhatnagar, S., Silver, D., Szepesvári, C., \& Wiewiora, E. (2009). Fast gradient-descent methods for temporal-difference learning with linear function approximation. In A. P. Danyluk, L. Bottou, \& M. L. Littman (Eds.), Proceedings of the twenty-sixth annual international conference on machine learning (ICML 2009) (pp. 993-1000). New York: ACM.

Suttorp, T., Hansen, N., \& Igel, C. (2009). Efficient covariance matrix update for variable metric evolution strategies. Machine Learning, 75(2), 167-197.

Szita, I., \& Lôrincz, A. (2006). Learning Tetris using the noisy cross-entropy method. Neural Computation, 18(12), 2936-2941.

Szita, I., \& Lórincz, A. (2007). Learning to play using low-complexity rule-based policies: Illustrations through Ms. Pac-Man. The Journal of Artificial Intelligence Research, 30, 659-684.

Szita, I., \& Lőrincz, A. (2008). The many faces of optimism: a unifying approach. In W. W. Cohen, A. McCallum, \& S. T. Roweis (Eds.), Proceedings of the twenty-fifth international conference on machine learning (ICML 2008) (pp. 1048-1055). New York: ACM.

Tedrake, R., Zhang, T. W., \& Seung, H. S. (2004). Stochastic policy gradient reinforcement learning on a simple 3D biped. In Proceedings of the 2004 IEEE/RSJ international conference on intelligent robots and systems (IROS 2004) (pp. 2849-2854). New York: IEEE Press.

Tesauro, G. (1992). Practical issues in temporal difference learning. Machine Learning, 8(3-4), 257-277.

Tesauro, G., Jong, N. K., Das, R., \& Bennani, M. N. (2007). On the use of hybrid reinforcement learning for autonomic resource allocation. Cluster Computing, 10(3), 287-299.

Thrun, S., \& Schwartz, A. (1993). Issues in using function approximation for reinforcement learning. In M. Mozer, P. Smolensky, D. Touretzky, J. Elman, \& A. Weigend (Eds.), Proceedings of the 1993 connectionist models summer school (pp. 255-263). Hillsdale: Lawrence Erlbaum.

Togelius, J., Schaul, T., Wierstra, D., Igel, C., Gomez, F., \& Schmidhuber, J. (2009). Ontogenetic and phylogenetic reinforcement learning. In Künstliche Intelligenz (pp. 30-33).

Tsitsiklis, J. N., \& Van Roy, B. (1997). An analysis of temporal-difference learning with function approximation. IEEE Transactions on Automatic Control, 42, 674-690.

van Seijen, H., van Hasselt, H., Whiteson, S., \& Wiering, M. (2009). A theoretical and empirical analysis of Expected Sarsa. In Proceedings of the 2009 IEEE symposium on adaptive dynamic programming and reinforcement learning (ADPRL 2009) (pp. 177-184). New York: IEEE Press.

Vilalta, R., \& Drissi, Y. (2002). A perspective view and survey of meta-learning. Artificial Intelligence Review, 18(2), 77-95.

Watkins, C. J. C. H. (1989). Learning from delayed rewards. Ph.D. thesis, King's College, Cambridge, UK.

Watkins, C. J. C. H., \& Dayan, P. (1992). Q-learning. Machine Learning, 8(3-4), 279-292.

Whitehead, S. D., \& Ballard, D. H. (1991). Learning to perceive and act by trial and error. Machine Learning, $7(1), 45-83$.

Whiteson, S., \& Stone, P. (2004). Adaptive job routing and scheduling. Engineering Applications of Artificial Intelligence, 17(7), 855-869. Special issue on Autonomic Computing and Automation.

Whiteson, S., \& Stone, P. (2006). Evolutionary function approximation for reinforcement learning. Journal of Machine Learning Research, 7, 877-917.

Whiteson, S., Tanner, B., Taylor, M. E., \& Stone, P. (2011). Protecting against evaluation overfitting in empirical reinforcement learning. In Proceedings of the 2011 IEEE Symposium on Adaptive Dynamic Programming and Reinforcement Learning (ADPRL 2011) (pp. 120-127). New York: IEEE Press. 
Whiteson, S., Taylor, M. E., \& Stone, P. (2010). Critical factors in the empirical performance of temporal difference and evolutionary methods for reinforcement learning. Autonomous Agents and Multi-Agent Systems, 21(1), 1-35.

Wolpert, D. H., \& Macready, W. G. (1997). No free lunch theorems for optimization. IEEE Transactions on Evolutionary Computation, 1(1), 67-82.

Xu, L., Hutter, F., Hoos, H. H., \& Leyton-Brown, K. (2008). SATzilla: portfolio-based algorithm selection for SAT. The Journal of Artificial Intelligence Research, 32, 565-606.

Zhang, W., \& Dietterich, T. G. (1995). A reinforcement learning approach to job-shop scheduling. In Proceedings of the fourteenth international joint conference on artificial intelligence (IJCAI 1995) (pp. 1114-1120). Los Altos: Kaufmann. 